\title{
Symmetric submanifolds of compact symmetric spaces
}

\author{
By
}

\author{
Hiroo NAITOH*
}

\section{Introduction}

This paper is the final report for the author's anouncement of the same title, appeared in Lect. Notes in Math., 1090, Springer-Verlag ([15]). It contains the results of the anouncement and their detailed proofs, and some further results.

Now symmetric submanifold is defined analogously to riemannian symmetric space. Namely, for riemannian symmetric space it is assumed, the existence of the (intrinsic) symmetry at each point. And for symmetric submanifold it is assumed, the existence of the extrinsic symmetry at each point in the submanifold. If the ambient spaces are riemannian symmetric spaces, symmetric submanifolds are locally characterized as submanifolds with parallel second fundamental form which satisfy some condition on the normal spaces. (See Theorem 1.3.) This characterization corresponds to the characterization that riemannian symmetric spaces are riemannian manifolds with parallel curvature tensor locally. If the ambient spaces are rank-one symmetric spaces, submanifolds with parallel second fundamental form have already been classified by several mathematicians. (See [1], [4], [5], [9], [10], [13], [14], [17], [18], [21], [22].). Hence we can take up symmetric submanifolds of their spaces. But if the ambient spaces are other riemannian symmetric spaces, the symmetric submanifolds are almost unknown except Tsukada [23]. In this paper we consider the classification for the case when the ambient spaces are compact simply connected riemannian symmetric spaces.

Firstly we will show that symmetric submanifolds of compact riemannian manifolds are equivariant for certain Lie groups acting transitively on the submanifolds, that is, the inclusions are induced from Lie group homomorphisms of the Lie groups into the isometry groups of the ambient spaces. (See Theorem 2.5.) This result implies that our classification may be reduced into that of certain algebraic objects associated with Lie group or Lie algebra.

Next for symmetric submanifolds we will define the totally geodesic symmetric submanifolds tangent to the original symmetric submanifolds, and divide our classification problem into the following two steps. The first step is to classify the associated totally geodesic symmetric submanifolds. This is reduced to the local classification of non-

(*) Partially supported by the Grant-in-aid for Scientic Research, No 60740043

Received July 12,1985 
compact affine symmetric spaces by Berger [2]. (See Theorem 3.3 and Proposition 3.6.) And the second step is to fix a totally geodesic symmetric submanifold and to find all symmetric submanifolds tangent to it. The classification is also reduced to that of certain algebraic objects associated with the corresponding non-compact affine symmetric space. (See Theorem 4.4 and Cororally 4.6.)

Lastly, by using this reduction, we will give a sufficient condition for totally geodesic symmetric submanifolds to satisfy that all symmetric submanifolds tangent to them are the original totally geodesic symmetric submanifolds. (See Theorem 5.2.) There are many examples satisfying this conditions. Moreover we will consider two series of totally geodesic symmetric submanifolds which don't satisfy this condition. The one is close to totally complex totally geodesic submanifolds of compact quaternionic symmetric spaces, and the other is close to totally real totally geodesic submanifolds of compact hermitian symmetric spaces. (See Theorem 5.4 and Theorem 5.7 .) In these cases the symmetric submanifolds tangent to them are not always totally geodesic.

\section{Symmetric immersions}

Let $S, M$ be connected riemannian manifolds and let $f$ be an isometric immersion of $S$ into $M$. Denote by $T_{p} S, N_{p} S$ the tangent space, the normal space at $p \in S$ respectively. The immersion $f$ is called symmetric if for each $p \in S$ there exist an isometry $s_{p}$ of $S$ and an isometry $t_{p}$ of $M$ such that

$$
\begin{aligned}
& t_{p} \circ f=f \circ s_{p}, \\
& s_{p}(p)=p \text { and thus } t_{\mathrm{p}}(f(p))=f(p), \\
& \left(t_{p}\right)_{*} f_{*}(X)=-f_{*}(X),\left(t_{p}\right)_{*} \xi=\xi
\end{aligned}
$$

for $X \in T_{p} S, \xi \in N_{p} S$. Note that $\left(s_{p}\right)_{*} X=-X$ for $X \in T_{p} S$ by (1.1), (1.3) and thus $S$ is a riemannian symmetric space. Here we call the isometries $s_{p}, t_{p}$ the intrinsic, the extrinsic symmetries at $p$ respectively. If $f$ is an imbedding, the image $f(S)$ is called a symmetric submanifold and is identified with the manifold $S$. Then the isometry $s_{p}$ is identified with the restriction of $t_{p}$ to $S$. Moreover if $S$ is regular, the restriction is automatically smooth without the existence of $s_{p}$. Hence the definition of regular symmetric submanifold may be rewritten in the following form. For each $p \in S$ there exists an isometry $t_{p}$ of $M$ such that

$$
\begin{aligned}
& t_{p}(p)=p, t_{p}(S)=S, \\
& \left(t_{p}\right) * X=-X,\left(t_{p}\right)_{*} \xi=\xi
\end{aligned}
$$

for $X \in T_{p} S, \xi \in N_{p} S$.

For the local versions of symmetric immersion, symmetric submanifold we assume only the existence of an isometry $s_{p}$ around $p$ and an isometry $t_{p}$ around $f(p)$ satisfying the conditions $(1.1) \sim(1.3)$ and call these a locally symmetric immersion, a locally symmetric submanifold respectively. Then the manifold $S$ is a locally riemannian symmetric space. 
Return to an isometric immersion $f$ of $S$ into $M$ and denote by $\alpha$ the second fundamental form of $f$ and by $\nabla^{*} \alpha$ the covariant derivative of $\alpha$ defined in the following.

$$
\left(\nabla_{X}^{*} \alpha\right)(Y, Z)=D_{X}(\alpha(Y, Z))-\alpha\left(\nabla_{X} Y, Z\right)-\alpha\left(Y, \nabla_{X} Z\right)
$$

for vector fields $X, Y, Z$ tangent to $S$. Here $D, \nabla$ denote the normal connection for $f$, the riemannian connection on $S$ respectively. The immersion $f$ is called parallel if $\nabla^{*} \alpha=0$ and moreover if $f$ is an imbedding, the image $f(S)$ or $S$ is called a parallel submanifold.

Denote by $\bar{R}$ the curvature tensor on $M$. A linear subspace $V$ in a tangent space of $M$ is called curvature-invariant if $\bar{R}(V, V) V \subset V$. If $f$ is a parallel immersion, the subspaces $f_{*}\left(T_{p} S\right), p \in S$, are curvature-invariant by the Coddazi equation for $f$.

Next assume that $f$ is a parallel immersion. Fix a point $p \in S$ and let $\lambda$ be a linear endomorphism of $T_{f(p)} M$ such that

$$
\lambda\left(f_{*}(X)\right)=-f_{*}(X), \lambda(\xi)=\xi
$$

for $X \in T_{p} S, \xi \in N_{p} S$. Let $\gamma(t),|t|<l$, be a geodesic in $S$ such that $\gamma(0)=p$. Then it follows the following

LEMMA 1.1. (Strübing [16]). Assume that $\Lambda$ is an isometry of $M$ defined on a domain containing the image $f(\gamma(t)),|t|<l$, and satisfying

$$
\Lambda(f(p))=f(p), \Lambda_{f(p)}=\lambda .
$$

Then it holds $\Lambda(f(\gamma(t)))=f(\gamma(-t))$ for all $t$ such that $|t|<l$.

Now if $f$ is a parallel immersion of $S$ into a locally riemannian symmetric space $M$, the manifold $S$ is also a locally riemannian symmetric space. Hence we may consider the relation between symmetric immersions and parallel immersions into locally riemannian symmetric spaces. Firstly we prepare the following

LEMMA 1.2. (cf. Helgason [6]). Let $L, L^{\prime}$ be locally riemannian symmetric spaces of the same dimension, furnished with the curvature tensors $R, R^{\prime}$ respectively. Fix $p \in L, p^{\prime} \in L^{\prime}$ and let $\lambda$ be a linear isometry of $T_{p} L$ onto $T_{p^{\prime}} L^{\prime}$ such that

$$
\lambda(R(X, Y) Z)=R^{\prime}(\lambda(X), \lambda(Y)) \lambda(Z)
$$

for $X, Y, Z \in T_{p} L$. Then,

(1) there exists a local isometry $\Lambda$ of a domain around ponto a domain around $p^{\prime}$ such that $\Lambda(p)=p^{\prime}, \Lambda_{* p}=\lambda$. If $L$ is simply connected and complete and if $L^{\prime}$ is complete, the isometry $\Lambda$ exists globally on $L$.

For the geodesic $\gamma(t)$ in $L$ such that $\gamma(0)=p, \dot{\gamma}(0)=X$, denote by $\gamma^{\lambda}(t)$ the geodesic in $L^{\prime}$ such that $\gamma^{\lambda}(0)=p^{\prime}, \dot{\gamma}^{\lambda}(0)=\lambda(X)$.

(2) Let $\Lambda$ be a (not necessarily smooth) mapping of a domain around $p$ into $L^{\prime}$ such that $\Lambda(\gamma(t))=\gamma^{\lambda}(t)$ for all local geodesics $\gamma(t)$ starting from $p$. Then $\Lambda$ is a unique smooth isometry 
on some domain around $p$ such that $\Lambda_{* p}=\lambda$. Moreover assume that $L, L^{\prime}$ are complete. If $\Lambda$ is defined globally on $L$, it is a smooth isometry on $L$.

THEOREM 1.3. Let $f$ be an isometric immersion of $S$ into a locally riemannian symmetric space $M$. Then $f$ is a locally symmetric immersion if and only if it is a parallel immersion such that the normal spaces $N_{p} S, p \in \mathrm{S}$, are curvature-invariant subspaces of $T_{f(p)} M$ respectively.

ProOF. Assume that $f$ is locally symmetric. Since $t_{p}, s_{p}$ are isometries such that $t_{p} \circ f=f \circ s_{p}$, we have

$$
\begin{aligned}
& \left(\nabla_{\left(s_{p}\right)_{\infty} X} \alpha\right)\left(\left(s_{p}\right)_{*} Y,\left(s_{p}\right)_{*} Z\right)=\left(t_{p}\right)_{*}\left(\left(\nabla_{X} \alpha\right)(Y, Z)\right), \\
& \bar{R}\left(\left(t_{p}\right)_{*} \xi,\left(t_{p}\right)_{*} \zeta\right)\left(t_{p}\right)_{*} \eta=\left(t_{p}\right)_{*}(\bar{R}(\xi, \zeta) \eta)
\end{aligned}
$$

for $X, Y, Z \in T_{p} S, \xi, \zeta, \eta \in N_{p} S$. Then, by (1.3), it follows

$$
\begin{aligned}
& -\left(\nabla_{X} \alpha\right)(Y, Z)=\left(\nabla_{X} \alpha\right)(Y, Z), \\
& \bar{R}(\xi, z) \eta=\left(t_{p}\right)_{*}(\bar{R}(\xi, \zeta) \eta) .
\end{aligned}
$$

These imply that $\nabla \alpha=0$ and $\bar{R}\left(N_{p} S, N_{p} S\right) N_{p} S \subset N_{p} S$ respectively.

Conversely assume that $f$ is a parallel immersion such that the normal spaces are curvature-invariant. Fix $p \in S$. Then the subspace $f_{*} T_{p} S$ is also curvature-invariant. Hence, by the property of the curvature tensor $\bar{R}$, it follows

$$
\left\{\begin{array}{l}
\bar{R}\left(f_{*} T_{p} S, f_{*} T_{p} S\right) f_{*} T_{p} S \subset f_{*} T_{p} S, \quad \bar{R}\left(f_{*} T_{p} S, f_{*} T_{p} S\right) N_{p} S \subset N_{p} S, \\
\bar{R}\left(f_{*} T_{p} S, N_{p} S\right) f_{*} T_{p} S \subset N_{p} S, \quad \bar{R}\left(f_{*} T_{p} S, N_{p} S\right) N_{p} S \subset f_{*} T_{p} S, \\
\bar{R}\left(N_{p} S, N_{p} S\right) f_{*} T_{p} S \subset f_{*} T_{p} S, \quad \bar{R}\left(N_{p} S, N_{p} S\right) N_{p} S \subset N_{p} S .
\end{array}\right.
$$

Define a linear isometry $\lambda$ of $T_{f(p)} M$ as in (1.5). Then the relations (1.7) imply that $\lambda$ satisfies (1.6). Hence there exists a local isometry $t_{p}$ around $f(p)$ such that $t_{p}(f(p))=f(p)$, $\left(t_{p}\right)_{\cdot f(p)}=\lambda$. Then, by Lemma 1.1, it holds $t_{p}(f(\gamma(t))=f(\gamma(-t))$ for all local geodesics $\gamma(t)$ in $S$ starting from $p$. Let $s_{p}$ be a mapping defined around $p$ such that $f \circ s_{p}=t_{p} \circ f$. Obviously it follows $s_{p}(\gamma(t))=\gamma(-t)$. By Lemma 1.2, (2), the mapping $s_{p}$ is a smooth isometry. It is obvious that these isometries $t_{p}, s_{p}$ satisfy the conditions (1.1) (1.3). Hence $f$ is locally symmetric.

q.e.d.

CORORALLY 1.4. Let $M$ be a simply connected riemannian symmetric space and let $S$ be a submanifold of $M$. Then $S$ is a symmetric submanifold if and only if it is a complete parallel submanifold such that the normal spaces are curvature-invariant.

Particularly a complete locally symmetric submanifold is always a symmetric submanifold.

PROOF. If $S$ is symmetric, it is a riemannian symmetric space and thus it is complete. By Theorem 1.3, it is a complete parallel submanifold such that the normal spaces are curvature-invariant.

Conversely assume that $S$ is such a submanifold. And recall the local isometries $t_{p}, s_{p}$ 
defined in the proof of Theorem 1.3. Since $M$ is simply connected and $S$ is complete, they are defined globally as smooth isometries by Lemma 1.2 . Hence $S$ is symmetric. q.e.d.

REMARK 1.5. Assume that $M$ has constant curvature and let $f$ be an isometric immersion of $S$ into $M$. Then the normal spaces are always curvature-invariant.

\section{The equivariance of symmetric immersions and their classification problem}

Let $S, M$ be riemannian manifolds. Denote by $I^{0}(S), I^{0}(M)$ the connected components of the isometry groups of $S, M$ respectively, containing the identity transformations. Then the Lie algebras $\mathrm{g}, \overline{\mathrm{g}}$ of $I^{0}(S), I^{0}(M)$ are canonically identified with the Lie algebras of all Killing vector fields on $S, M$ respectively.

Let $f$ be an isometric immersion of $S$ into $M$. Define a Lie subalgebra $\mathfrak{g}_{f}$ of $\mathfrak{g}$ as follows. A Killing vector field $X$ on $S$ is contained in $g_{f}$ if and only if it satisfies $f_{*} X=\bar{X} \circ f$ for some $\bar{X} \in \overline{\mathfrak{g}}$. Let $\mathfrak{h}$ be a Lie subalgebra of $\mathfrak{g}_{f}$. Then $\mathfrak{h}$ is called an $f_{*}$-related subalgebra if there exists a Lie algebra homomorphism $\rho$ of $\mathfrak{h}$ into $\bar{g}$ such that $f_{*} X=\rho(X) \circ f$ for $X \in \mathfrak{h}$. Such a $\rho$ is called an $f_{*}$-related Lie homomorphism.

Let $\mathfrak{h}$ be an $f_{*}$-related subalgebra and $\rho$ an $f_{*}$-related Lie homomorphism of $\mathfrak{h}$ into $\overline{\mathfrak{g}}$. Denote by $H$ the universal convering of the connected Lie subgroup of $I^{0}(S)$ with Lie algebra $\mathfrak{h}$, and by the same notation $\rho$ the Lie group homomorphism of $H$ into $I^{0}(M)$ whose differential is $\rho$. The Lie group $H$ acts on $S$ through $I^{0}(S)$, while it acts on $M$ through $\rho(H)$ $\subset I^{0}(M)$.

LEMMA 2.1. The isometric immersion $f$ is H-equivariant, i.e.,

$$
\rho(h)(f(q))=f(h(q))
$$

for $h \in H, q \in S$.

Proof. Let $X \in \mathfrak{h}$ and fix $q \in S$. We may show that

$$
\rho(\exp t X)(f(q))=f((\exp t X)(q))
$$

for the one parameter subgroup $\exp t X$ of $H$. Note that $\rho(\exp t X)(f(q)),(\exp t X)(q)$ are integral curves of Killing vector fields $\rho(X), X$ respectively. Since $f_{*}(X)=\rho(X) \circ f$, the curve $f((\exp t X)(q))$ is also the integral curve of $\rho(X)$ through $q$ at $t=0$. Hence the equality (2.1) holds by the uniqueness of integral curves.

q.e.d.

Now let $f$ be a symmetric immersion of $S$ into a riemannian manifold $M$. Recall that $S$ is a riemannian symmetric space. Let $\gamma(t),-\infty<t<\infty$, be a complete geodesic in $S$. Then it follows

$$
s_{\gamma(c)}(\gamma(t))=\gamma(2 c-t)
$$

for $c, t \in \boldsymbol{R}$. Denote by $\tau_{a, b}, a \leqq b$, the parallel translation for the riemannian connection on $S$, from $T_{\gamma(a)} S$ to $T_{\nu(b)} S$ along $\gamma(t)$. Then the following equalities hold: 


$$
\begin{aligned}
& \tau_{a, b}=-\left(s_{\gamma(a+b / 2)}\right)_{* \gamma(a),}, \\
& s_{\gamma(a)} \circ s_{\gamma(0)}{ }^{\circ} s_{\gamma(b)}=s_{\gamma(a+b) .} .
\end{aligned}
$$

Let $S(t)$ be a curve in $I^{0}(S)$ defined by

$$
S(t)=s_{\gamma(t / 2)}{ }^{\circ} s_{\gamma(0)}
$$

for all $t \in \boldsymbol{R}$. Then the equality (2.4) implies that $S(t)$ is a one parameter subgroup. (See Helgason [6] for (2.3), (2.4).)

Now consider the image curve $f(\gamma(t))$ of a complete geodesic $\gamma(t)$ in $S$. Denote by $\bar{\tau}_{a, b}$ the parallel translation for the normal connection, from $N_{\gamma(a)} S$ to $N_{\gamma(b)} S$ along $f(\gamma(t))$. Then we have the following lemma analogous to (2.3), (2.4). The proof is also along Helgason's arguements.

LEMMA 2.2. It holds that

$$
\bar{\tau}_{a, b}(\xi)=\left(t_{\gamma(a+b / 2)}\right)_{* f(\gamma(a))}(\xi)
$$

for $\xi \in \mathrm{N}_{\gamma(a)} S$ and moreover

$$
t_{\gamma(a)} \circ t_{\gamma(0)} \circ t_{\gamma(b)}=t_{\gamma(a+b)}
$$

Proof. Firstly we show the equality (2.5). Obviously it follows

$$
t_{\gamma(c)}(f(\gamma(t)))=f(\gamma(2 c-t)),\left(t_{\gamma(c)}\right) * N_{\gamma(t)} S=N_{\gamma(2 c-t)} S
$$

by (1.1), (2.2). Put $c=(\mathrm{a}+\mathrm{b}) / 2$ and fix $\xi \in N_{\gamma(a)} S$. Then normal vectors $\bar{\tau}_{a, c}(\xi), \xi$ are parallel and thus $\left(t_{\gamma(c)}\right)_{*}\left(\bar{\tau}_{a, c}(\xi)\right),\left(t_{\gamma(c)}\right) * \xi$ are also parallel by (2.7). Since $\left(t_{\gamma(c)}\right) *\left(\bar{\tau}_{a, c}(\xi)\right)=\bar{\tau}_{a, c}(\xi)$ and $\bar{\tau}_{a, c}(\xi)$ is parallel to $\bar{\tau}_{a, b}(\xi)$, normal vectors $\bar{\tau}_{a, b}(\xi),\left(t_{\gamma(c)}\right) * \xi$ are parallel. Hence it follows that $\bar{\tau}_{a, b}(\xi)=\left(t_{\gamma(c)}\right) * \xi$.

Next we show the equality (2.6). Put $\psi=t_{\gamma(a)} \circ t_{\gamma(0)} \circ t_{\gamma(b)}$ and $p=\gamma(a+b)$. Then it follows $\psi(f(p))=t_{p}\left(f(p)=f(p)\right.$ by (2.7). Consider the differential $\psi_{* f(p)}$ of $\psi$ at $f(p)$. The restriction of $\psi_{* f(p)}$ to $f_{*}\left(T_{p} S\right)$ is the minus identity by (1.1), (2.4), and moreover that of $\psi_{* f(p)}$ to $N_{p} S$ is the identity by (2.5). Hence it follows that $\psi_{* f(p)}=\left(t_{p}\right)_{*_{f(p)}}$. Since $\psi, t_{p}$ are isometries, it holds $\psi=t_{p}$ on $M$.

q.e.d.

Let $T(t)$ be a curve in $I^{0}(M)$ defined by

$$
T(t)=t_{\gamma(t / 2)} \circ t_{\gamma(0)}
$$

for all $t \in \boldsymbol{R}$. Then the equality (2.6) implies that $T(t)$ is a one parameter subgroup.

Now fix $p \in S$ and define an involutive automorphism $\delta$ of $I^{0}(S)$ by $\delta(g)=s_{p} \circ g \circ s_{p}$ for $g \in I^{0}(S)$. Then the differential induces an involutive automorphism of $g$. Denote it by the same notation $\delta$. Let $\mathfrak{p}$ be the $(-1)$-eigenspace of $\delta$. Let $X \in \mathfrak{p}$. Then the integral curve $\gamma(t)$ of $X$ such that $\gamma(0)=p$ is a complete geodesic in $S$. Moreover the one parameter subgroup $S(t)$ defined from $\gamma(t)$ coincides with the one parameter subgroup exp $t X$ generated by $X$. Put $\mathfrak{t}_{0}=[\mathfrak{p}, \mathfrak{p}]$ and $\mathfrak{g}_{0}=\mathfrak{t}_{0} \oplus \mathfrak{p}$. 
PROPOSITION 2.3. Let $f$ be a symmetric immersion of $S$ into a riemannian manifold $M$. Then it holds $\mathrm{g}_{0} \subset \mathrm{g}_{f}$.

Proof. We may show that $\mathfrak{p} \subset g_{f}$. Take $X \in \mathfrak{p}$ and let $\gamma(t)$ be the integral complete geodesic such that $\gamma(0)=p$. Then the one parameter subgroups $S(t), T(t)$ satisfy $T(t) \circ f$ $=f \circ S(t)$ by (1.1). Let $\bar{X}$ be the Killing vector field on $M$ which generates $T(t)$. Since $S(t)$ is generated by $X$, it follows $f_{*}(X)=\bar{X} \circ f$ and thus $X \in g_{f}$. This implies that $\mathfrak{p} \subset g_{f}$. q.e.d.

Next we consider a sufficient condition for $g_{0}$ to be $f_{*}$-related. Denote by $\bar{K}$ the Lie subgroup of $I^{0}(M)$ whose elements leave the point $f(p)$ fixed and by $\bar{f}$ its Lie algebra. Let $\overline{\mathfrak{g}}_{0} \subset \bar{g}$ be the Lie subalgebra of Killing vector fields $\bar{X}$ on $M$ such that $f_{*}(X)=\bar{X} \circ f$ for some $X \in \mathfrak{g}_{0}$. Define the projection $\pi$ of $\overline{\mathrm{g}}_{0}$ onto $\mathrm{g}_{0}$ by $\pi(\bar{X})=X$ for $\bar{X} \in \overline{\mathrm{g}}_{0}$, where $f_{*}(X)=\bar{X} \circ f$. Since $f$ is an immersion, the projection is a well-defined Lie homomorphism.

LEMMA 2.4. If $\overline{\mathfrak{g}}$ admits a nondegenerate symmetric bilinear form $\bar{B}$ satisfying the following conditions, then $g_{0}$ is an $f_{*}$-related subalgebra of $g_{f}$. The conditions are:

(i) endomorphisms ad $(X), X \in \overline{\mathrm{g}}_{0}$, of $\overline{\mathrm{g}}_{0}$ are skew adjoint with respect to $\bar{B}$, i.e.,

$$
\bar{B}(\operatorname{ad}(\bar{X}) \bar{Y}, \bar{Z})+\bar{B}(\bar{Y}, \text { ad }(\bar{X}) \bar{Z})=0
$$

for $\bar{Y}, \bar{Z} \in \bar{g}_{0}$, and

(ii) the restriction of $\bar{B}$ to $\bar{f}$ is positive (or negative) definite.

ProOF. Note that the kernel $\operatorname{Ker} \pi$ of $\pi$ is contained in $\overline{\mathfrak{l}}$. Then $\operatorname{Ker} \pi$ is a nondegenerate subspace in $\bar{g}$ with respect to $\bar{B}$ by (ii). Hence we have the direct sum $\overline{\mathfrak{g}}=\overline{\mathfrak{a}} \oplus \operatorname{Ker} \pi$, where $\overline{\mathfrak{a}}=\{\overline{\mathrm{X}} \in \overline{\mathfrak{g}} ; \bar{B}(\bar{X}, \operatorname{Ker} \pi)=\{0\}\}$. Put $(\operatorname{Ker} \pi)^{\perp}=\overline{\mathfrak{a}} \cap \overline{\mathfrak{g}}_{0}$. Then we also have the orthogonal direct sum $\overline{\mathrm{g}}_{0}=\operatorname{Ker} \pi \oplus(\operatorname{Ker} \pi)^{\perp}$. Since $\operatorname{Ker} \pi$ is an ideal of $\bar{g}_{0}$, the subspace $(\operatorname{Ker} \pi)^{\perp}$ is a Lie subalgebra of $\bar{g}_{0}$ by (i). Then the projection $\pi$ induces a Lie algebra isomorphism of $(\operatorname{Ker} \pi)^{\perp}$ onto $g_{0}$ and the inverse gives an $f_{*}$-related Lie homomorphism of $g_{0}$. Hence $g_{0}$ is $f_{*}$-related.

q.e.d.

Let $G_{0}$ be the universal covering of the connected Lie subgroup of $I^{0}(S)$ with Lie algebra $g_{0}$. Since $S$ is a riemannian symmetric space, the Lie group $G_{0}$ acts transitively on $S$.

THEOREM 2.5. Let $M$ be a riemannian manifold satisfying either of the followings:

(1) $I^{0}(M)$ is compact.

(2) $I^{0}(M)$ is semi-simple.

Then every symmetric immersion of $S$ into $M$ is $G_{0}$-equivariant.

Proof. Assume that $M$ satisfies (1). Then $\bar{g}$ admits a positive definite inner product such that ad $(\bar{X}), \bar{X} \in \bar{g}$, are skew symmetric. This inner product satisfies the conditions (i), (ii) in Lemma 2.4 . 
Next assume that $M$ satisfies (2). Take the Killing form of $\bar{g}$. Then it satisfies the conditions (i), (ii). (See Kobayashi-Nomizu [8], Vol. II, p. 247 for the proof.)

Hence, by Lemma 2.4, every symmetric immersion of $S$ into $M$ is $G_{0}$-equivariant.q.e.d.

REMARK 2.6. Compact riemannian manifolds satisfy the condition (1). And riemannian symmetric spaces of noncompact type satisfy the condition (2).

REMARK 2.7. Naitoh [12] has shown this Theorem for the case when $S$ are $n$-dimensional totally real symmetric submanifolds of the complex projective $n$-space, and Tsukada [21] for the case when $S$ are $n$-dimensional totally complex symmetric submanifolds of the quaternionic projective $n$-space. The crucial points of their arguements are the concrete constructions of $f_{*}$-related Lie homomorphisms.

CORORALLY 2.8. Let $M$ be a riemannian manifold satisfying either of the conditions (1), (2) in Theorem 2.5, and let $f$ be a symmetric immersion of $S$ into $M$. Then the image $f(S)$ has the structure of symmetric submanifold such that $f$ is a riemannian covering of $S$ onto $f(S)$.

PROOF. Let $\rho$ be an $f_{*}$-related Lie homomorphism of $g_{0}$ and denote by the same notation the Lie group homomorphism of $G_{0}$ to $I^{0}(M)$ whose differential is $\rho$. By Theorem 2.5 the image $f(S)$ is the $\rho\left(G_{0}\right)$-orbit at the fixed point $f(p)$. Since $\rho\left(G_{0}\right)$ has the structure of Lie subgroup such that $\rho$ is a Lie group homomorphism of $G_{0}$ onto $\rho\left(G_{0}\right)$, the image $f(S)$ has the differentiable structure as $\rho\left(G_{0}\right)$-orbit. Then $f(S)$ is a submanifold of $M$ and $f$ induces a smooth mapping of $S$ onto $f(S)$. Consider the metric on $f(S)$ induced from that on $M$. Since $S$ is complete, $f$ is a covering of $S$ onto $f(S)$.

Let $\bar{q} \in f(S)$ and take $q \in S$ such that $f(q)=\bar{q}$. Then, since $f(S)$ is a complete locally riemannian symmetric space, the restriction of $t_{q}$ to $f(S)$ gives the smooth intrinsic symmetry at $\bar{q}$ by Lemma 1.2 , (2). Hence $f(S)$ is a symmetric submanifold of $M$. q.e.d.

Let $f, f^{\prime}$ be isometric immersions of riemannian manifolds $S, S^{\prime}$ into riemannian manifolds $M, M^{\prime}$ respectively. The immersions $f, f^{\prime}$ are called equivalent to each other if there exist an isometry $\psi$ of $S$ onto $S^{\prime}$ and an isometry $\bar{\psi}$ of $M$ onto $M^{\prime}$ such that $f^{\prime} \circ \psi=$ $\bar{\psi} \circ f$ on $S$.

REMARK 2.9. Let $M$ be a riemannian manifold satisfying either of the conditions (1), (2) in Theorem 2.5, and let $f, f^{\prime}$ be symmetric immersions of simply connected riemannian manifolds $S, S^{\prime}$ into $M$. Then $f$ is equivalent to $f^{\prime}$ if and only if $f(S) \rightarrow M$ is equivalent to $f^{\prime}\left(S^{\prime}\right) \rightarrow M$.

In the rest of this section we consider the classification problem of symmetric submanifolds in riemannian symmetric spaces without euclidean factor. Since such riemannian symmetric spaces satisfy the condition (2) in Theorem 2.5 , it is natural to consider the problem. And the classification problem of symmetric immersions is also reduced to the 
problem by Remark 2.9.

Let $f$ be a symmetric immersion of $S$ into a riemannian symmetric space $M$. Let $p \in S$. Since $f_{*} T_{p} S$ is curvature-invariant, there exists a unique complete totally geodesic submanifold $N(p)$ such that $N(p) \ni f(p), T_{f(p)} N(p)=f_{*} T_{p} S$ (cf. Helgason [6]).

LEMMA 2.10. The submanifold $N(p)$ is symmetric. Moreover all symmetric submanifolds $N(p), p \in S$, are equivalent to one another.

Proof. Let $p^{\prime} \in N(p)$ and take a geodesic $\gamma(t)$ in $N(p)$ such that $\gamma(a)=p, \gamma(-a)=p^{\prime}$. Put $m=\gamma(0)$ and consider the geodesic symmetry $\bar{s}_{m}$ of $M$ at $m$. Then it holds $\bar{s}_{m}(p)=p^{\prime}$, and moreover the restriction of $\bar{s}_{m}$ to $N(p)$ induces an isometry of $N(p)$ by Lemma 1.2, (2). Hence it follows $\left(\bar{s}_{m}\right) * N_{p}(N(p))=N_{p^{\prime}}(N(p))$. Since $N_{p}(N(p))=N_{p} S$ is curvature-invariant, so is $N_{p^{\prime}}(N(p))$. Hence $N(p)$ is a symmetric submanifold by Theorem 1.3.

Next let $N(q)$ be the totally geodesic symmetric submanifold defined at $q \in S$. We show that $N(q)$ is equivalent to $N(p)$. Let $\gamma(t)$ be a geodesic in $S$ such that $\gamma(b)=p, \gamma(-b)=q$, and put $n=\gamma(0)$. Then the extrinsic symmetry $t_{n}$ satisfies that $\left(t_{n}\right)_{*} T_{p} N(p)=T_{q} N(q)$. Since $N(p), N(q)$ are totally geodesic, the restriction of $t_{n}$ to $N(p)$ induces an isometry of $N(p)$ onto $N(q)$ by Lemma $1.2,(2)$. Hence $N(q)$ is equivalent to $N(p)$.

q. e. d.

Hereafter this $N(p)$ is denoted simply by $N$ and is called the totally geodesic symmetric submanifold associated with $f$.

REMARK 2.11. Let $f, f^{\prime}$ be symmetric immersions into riemannian symmetric spaces $M, M^{\prime}$ and $N, N^{\prime}$ the totally geodesic symmetric submanifolds associated with $f, f^{\prime}$ respectively. If $f$ is equivalent to $f^{\prime}, N$ is also equivalent to $N^{\prime}$. (See the last arguement of the proof for Lemma 2.10.)

Next let $M=M_{1} \times M_{2}$ be the riemannian product of riemannian manifolds $M_{1}, M_{2}$. Let $f, \hat{f}$ be isometric immersions of $S$ to $M, M_{1}$ respectively and let $m_{2}$ be a point in $M_{2}$. Moreover assume that $f(q)=\left(\hat{f}(q), m_{2}\right)$ for $q \in S$. Then $f$ is symmetric if and only if $\hat{f}$ is symmetric. An isometric immersion $f$ of $S$ to $M$ is called substantial if for no proper riemannian product $M=M_{1} \times M_{2}$ there exist an isometric immersion $\hat{f}$ and a point $m_{2}$ satisfying the above assumption.

LEMMA 2.12. Assume that $M$ is a simply connected riemmanian symmetric space without euclidean factor. Let $f$ be a symmetric immersion of $S$ to $M$ and $N$ the totally geodesic symmetric submanifold associated with $f$. Then $f$ is substantial if and only if the inclusion of $N$ to $M$ is substantial.

Proof. Obviously $f$ is substantial if the inclusion of $N$ to $M$ is substantial. We show the converse. Fix $p \in S$ and set $N=N(p)$. Assume that the inclusion of $N$ to $M$ is not substantial, i.e., there exists a proper decomposition $M=M_{1} \times M_{2}$ such that $N \subset M_{1} \times\left\{m_{2}\right\}$ 
for some $m_{2} \in M_{2}$. Let $q \in S$ and take a geodesic $\gamma(t)$ in $S$ such that $\gamma(0)=p, \gamma(a)=q$. Then it holds that $(T(a))_{*} f_{*} T_{p} S=f_{*} T_{q} S$. Let $T M=T M_{1} \oplus T M_{2}$ be the decomposition of the tangent bundle $T M$ associated with the product decomposition $M=M_{1} \times M_{2}$. Since $T(a)$ $\in I^{0}(M)=I^{0}\left(M_{1}\right) \times I^{0}\left(M_{2}\right)$, it follows $(T(a))_{*} T_{f(p)} M_{1}=T_{f(q)} M_{1}$. Moreover since $f_{*} T_{p} S=$ $T_{f(p)} N \subset T_{f(p)} M_{1}$ by the assumption, it follows $f_{*} T_{q} S \subset T_{f(q)} M_{1}$. Hence we have $f(S)$ $\subset M_{1} \times\left\{m_{2}\right\}$. This implies that $f$ is not substantial. Consequently the inclusion of $N$ to $M$ is substantial if $f$ is substantial.

q.e.d.

Now we propose the following

PROBLEM. Classify the substantial symmetric submanifolds of simply connected riemannian symmetric spaces without euclidean factor, up to the equivalence.

By the virture of Remark 2.11, Lemma 2.12, this problem is devided into the following two steps.

The First Step. Classify the substantial totally geodesic symmetric submanifolds.

The Second Step. Next fix a substantial totally geodesic symmetric submanifold. And classify the symmetric submanifolds which have it as associated totally geodesic symmetric submanifold.

In the following sections we will study this problem when the ambient spaces are compact simply connected riemannian symmetric spaces.

\section{Totally geodesic symmetric submanifolds}

A symmetric Lie algebra is, by definition, a pair $(g, \theta)$ of Lie algebra $g$ and an involutive automorphism $\theta$ on $\mathfrak{g}$. Let $\mathfrak{g}=\mathfrak{f} \oplus \mathfrak{p}$ be the eigenspace decomposition by $\theta$ into the $(+1)$ eigenspace $\mathfrak{t}$ and the $(-1)$-eigenspace $\mathfrak{p}$. Then the symmetric Lie algebra $(\mathfrak{g}, \theta)$ is called effective if the adjoint representation $\operatorname{ad}_{\mathfrak{p}}$ of $\mathfrak{f}$ into $\mathrm{gl}(\mathfrak{p})$ is faithful, where $\mathfrak{g l}(\mathfrak{p})$ denotes the Lie algebra of all endomorphisms of $\mathfrak{p}$. Next a quadruple $(\mathfrak{g}, \sigma, \tau,\langle>)$ satisfying the following conditions is called a compact orthogonal quadruple, abbreviated as COQ: (1) $g$ is a Lie algebra of compact type, namely compact semisimple Lie algebra, $(2)(\mathfrak{g}, \sigma),(\mathfrak{g}, \tau)$ are symmetric Lie algebras, (3) $\sigma \circ \tau=\tau \circ \sigma$, and (4) $\langle>$ is a positive definite inner product on $g$ which is left invariant by $\sigma, \tau$ and for which $\operatorname{ad}_{\mathfrak{g}}(X), X \in \mathfrak{g}$, are skew symmetric. A COQ $(\mathfrak{g}, \sigma, \tau,\langle>)$ is called effective if the symmetric Lie algebras $(\mathfrak{g}, \sigma),(\mathfrak{g}, \tau)$ are effective. And two COQ's $\left(g, \sigma, \tau,\langle>),\left(g^{\prime}, \sigma^{\prime}, \tau^{\prime},\left\langle>^{\prime}\right)\right.\right.$ are called equivalent to each other if there exists a Lie algebra isomorphism $\phi$ of $g$ onto $g^{\prime}$ such that $\phi \circ \sigma=\sigma^{\prime} \circ \phi, \phi \circ \tau=\tau^{\prime} \circ \phi$, and $\langle\phi(X), \phi(Y)\rangle^{\prime}=\langle X, Y\rangle$ for $X, Y \in g$. Define the "direct sum" of finite COQ's canonically. Then the direct sum keeps the equivalence and moreover it is effective if and only if each is effective.

Let $M$ be a compact simply connected riemannian symmetric space and $N$ a substantial totally geodesic symmetric submanifold of $M$. Hereafter this pair is denoted by $(M, N)$. 
Firstly we note that the Lie algebra $\bar{g}$ of $I^{0}(M)$ is of compact type. Fix $p \in N$ and denote by $\bar{s}_{p}$ the geodesic symmetry of $M$ at $p$. Then $\bar{s}_{p}, t_{p}$ induce involutive automorphisms $\sigma, \tau$ of $I^{0}(M)$ defined by

$$
\sigma(g)=\bar{s}_{p} \circ g \circ \bar{s}_{p}, \tau(g)=t_{p} \circ g \circ t_{p}
$$

for $g \in I^{0}(M)$. The differentials are also denoted by the same notations. Since $\left[\bar{s}_{p}, t_{p}\right]=0$, it follows

$$
[\sigma, \tau]=0 .
$$

Let $\overline{\mathfrak{g}}=\overline{\mathfrak{f}} \oplus \overline{\mathrm{p}}$ be the eigenspace decomposition by $\sigma$ into the $(+1)$-eigenspace $\overline{\mathfrak{f}}$ and the (-1)-eigenspace $\overline{\mathfrak{p}}$. Identify $\overline{\mathfrak{p}}$ with $T_{p} M$ by the identification: $X \leftrightarrow X_{p}$ for $X \in \overline{\mathfrak{p}}$. Then the restriction of the riemannian metric to $T_{p} M$ induces an inner product \langle\rangle$_{\bar{p}}$ on $\bar{p}$ such that $\operatorname{ad}_{\overline{\mathfrak{p}}}(T), T \in \overline{\mathfrak{f}}$, are skew symmetric. Note that $(\overline{\mathfrak{g}}, \sigma)$ is effective and thus

$$
[\overline{\mathfrak{p}}, \overline{\mathfrak{p}}]=\overline{\mathbf{f}} \text {. }
$$

Then the inner product \langle\rangle$_{\bar{p}}$ is uniquely extended to a positive definite inner product $\langle>$ on $\bar{g}$ which is left by $\sigma$ and for which $\operatorname{ad}_{\overline{\mathfrak{g}}}(X), X \in \overline{\mathfrak{g}}$, are skew symmetric.

LEMMA 3.1. The quadruple $(\overline{\mathrm{g}}, \sigma, \tau,<>)$ is an effective COQ and is independent of the fixed point $p$.

ProOF. Firstly we show that $(\overline{\mathfrak{g}}, \tau)$ is effective. Let $\overline{\mathfrak{g}}=\overline{\mathfrak{f}}^{*} \oplus \overline{\mathfrak{p}}^{*}$ be the eigenspace decomposition by $\tau$ into the (+1)-eigenspace $\overline{\mathfrak{f}}^{*}$ and the $(-1)$-eigenspace $\overline{\mathfrak{p}}^{*}$ and $\overline{\mathfrak{g}}_{1}$ the kernel of the adjoint representation $\operatorname{ad}_{\overline{\mathfrak{p}}^{*}}$ of $\overline{\mathfrak{f}}^{*}$ into $\operatorname{gl}\left(\overline{\mathfrak{p}}^{*}\right)$. Then $\overline{\mathfrak{g}}_{1}$ is an ideal of $\overline{\mathfrak{g}}$. Since $\sigma$ leaves $\overline{\mathfrak{f}}^{*}, \overline{\mathfrak{p}}^{*}$ invariant by (3.1), it also leaves $\overline{\mathfrak{g}}_{1}$ invariant. We suppose that $(\overline{\mathfrak{g}}, \tau)$ is not effective, i.e., $\overline{\mathfrak{g}}_{1} \neq\{0\}$. Moreover we may suppose that $\overline{\mathfrak{g}}_{1} \neq \overline{\mathrm{g}}$. If $\overline{\mathfrak{g}}_{1}=\overline{\mathfrak{g}}$, it follows $\tau=1_{\overline{\mathrm{g}}}$ and thus $t_{p}=1_{\mathrm{M}}$. This implies that $N=\{p\}$. Now let $\overline{\mathrm{g}}_{2}$ be the orthogonal compliment of $\overline{\mathrm{g}}_{1}$. Since $\overline{\mathrm{g}}_{2}$ is also left invariant by $\sigma$, the decomposition $\overline{\mathfrak{g}}=\overline{\mathfrak{g}}_{1} \oplus \overline{\mathfrak{g}}_{2}$ induces the proper riemannian product decomposition $M=M_{1} \times M_{2}$. Set $M_{1}(p)=\left(M_{1}, m_{2}\right), M_{2}(p)=\left(m_{1}, M_{2}\right)$, where $p=$ $\left(m_{1}, m_{2}\right)$. Since $t_{p}((\exp t X)(p))=(\exp t X)(p)$ for $X \in \bar{g}_{1}$, it follows that $T_{p}\left(M_{1}(p)\right) \subset N_{p} N$ and thus $T_{p} N \subset T_{p}\left(M_{2}(p)\right)$. Moreover since $N, M_{2}(p)$ are totally geodesic, it holds $N \subset M_{2}(p)$. This contradicts that $N \rightarrow M$ is substantial. Hence $(\overline{\mathfrak{g}}, \tau)$ is effective.

Next we show that $\tau$ leaves $\left\langle>\right.$ invariant. Note that \langle\rangle$_{\tilde{p}}$ is left invariant by $\tau$. Since $\tau$ leaves $\overline{\mathfrak{f}}, \overline{\mathrm{p}}$ invariant by (3.1), we may show that $\langle\tau(T), \tau(S)\rangle=\langle T, S\rangle$ for $T, S \in \overline{\mathrm{f}}$. By (3.2) we may put $T=[X, Y]$ for some $X, Y \in \bar{p}$. Then it follows

$$
\begin{aligned}
\langle\tau([X, Y]), \tau(S)\rangle & =-\langle\tau(Y), \tau([X, S])\rangle_{\bar{p}} \\
& =-\langle Y,[X, S]\rangle_{\bar{p}}=\langle[X, Y], S\rangle .
\end{aligned}
$$

Hence $\tau$ leaves $\langle>$ invariant.

Lastly we show the second claim. Let $q \in S$ and take a geodesic $\gamma(t)$ in $S$ such that $\gamma(0)$ $=p, \gamma(a)=q$. Then the isometry $T(a)$ induces a Lie group isomorphism $\phi$ of $I^{0}(M)$ defined 
by $\phi(g)=T(a) \circ g \circ T(a)^{-1}$ for $g \in I^{0}(M)$. The differential is also denoted by the same notation. It is obvious that this isomorphism of $\bar{g}$ gives an isomorphism between the effective COQ's defined at $p, q$ respectively.

q. e. d.

Define the "direct product" of finite submanifolds canonically. Then the direct product is a (substantial) symmetric submanifold if and only if each is a (substantial) symmetric submanifold. Our correspondence: $(M, N) \rightarrow(\overline{\mathfrak{g}}, \sigma, \tau,\langle\rangle)$ keeps each equivalence and translates the direct product of substantial totally geodesic symmetric submanifolds into the direct sum of effective COQ's.

Conversely let $(\overline{\mathfrak{g}}, \sigma, \tau,\langle\rangle)$ be an effective COQ and $\overline{\mathfrak{g}}=\overline{\mathfrak{f}} \oplus \overline{\mathfrak{p}}$ the eigenspace decomposition by $\sigma$. Take a compact simply connected Lie group $\bar{G}$ with Lie algebra $\bar{g}$ and the connected compact Lie subgroup $\bar{K}$ of $\bar{G}$ with Lie algebra $\bar{f}$. Then $M=\bar{G} / \bar{K}$ is a compact simply connected riemannian symmetric space, together with the $\bar{G}$-invariant metric induced from the restriction \langle\rangle$_{\bar{p}}$ of $\left\langle>\right.$ to $\bar{p}$. Here the tangent space $T_{o} M$ at $o=\bar{K}$ is identified with $\overline{\mathfrak{p}}$ by the identification: $\left.\overline{\mathrm{p}} \ni X \leftrightarrow(\mathrm{d} / \mathrm{dt})\right|_{t=0}(\exp t X)(o) \in T_{o} M$. Then the curvature tensor $\bar{R}_{o}$ at $o$ is identified as follows:

$$
\bar{R}_{o}(X, Y) Z=-\operatorname{ad}_{\bar{p}}([X, Y])(Z)
$$

for $X, Y, Z \in \overline{\mathfrak{p}}$. Note that $\tau$ leaves $\overline{\mathfrak{p}}$ invariant and let $\overline{\mathfrak{p}}_{ \pm}$be the $( \pm 1)$-eigenspaces of the restriction of $\tau$ to $\bar{p}$ respectively. Obviously $\bar{p}_{ \pm}$are curvature-invariant subspaces in $T_{o} M$ by (3.3). Let $N$ be the complete totally geodesic submanifold of $M$ such that $N \ni o, T_{o} N=\bar{p}_{-}$, $N_{o} N=\overline{\mathfrak{p}}_{+}$.

LEMMA 3.2. The totally geodesic submanifold $N$ is a substantial symmetric submanifold of $M$.

Proof. It is proved in the same way as in Lemma 2.10 that $N$ is symmetric. We show that $N$ is substantial. Suppose that $N$ is not substantial, i.e., there exists a proper product decomposition $M=M_{1} \times M_{2}$ such that $N \subset\left(M_{1}, o_{2}\right)$, where $o=\left(o_{1}, o_{2}\right)$. Let $\bar{p}_{1}, \bar{p}_{2}$ be the subspaces of $\bar{p}$ identified with the tangent spaces of $\left(M_{1}, o_{2}\right),\left(o_{1}, M_{2}\right)$ at $o$ respectively. Put $\overline{\mathfrak{g}}_{i}=\left[\overline{\mathfrak{p}}_{i}, \overline{\mathfrak{p}}_{i}\right] \oplus \overline{\mathfrak{p}}_{i}, i=1,2$. Since $\overline{\mathfrak{f}}=[\overline{\mathfrak{p}}, \overline{\mathfrak{p}}]$ by the effectivity of $(\overline{\mathfrak{g}}, \sigma)$, we have the ideal decomposition $\overline{\mathfrak{g}}=\overline{\mathfrak{g}}_{1} \oplus \overline{\mathrm{g}}_{2}$. Then, since $\overline{\mathfrak{p}}_{-} \subset \overline{\mathfrak{g}}_{1}$ by the assumption, the $(+1)$-eigenspace of $\tau$ contains $\overline{\mathfrak{p}}_{2}$ and thus $\overline{\mathrm{g}}_{2}$. This implies that the $(-1)$-eigenspace of $\tau$ is contained in $\overline{\mathrm{g}}_{1}$. This contradicts to the effectivity of $(\overline{\mathfrak{g}}, \tau)$. Hence $N$ is substantial. q.e.d.

Our correspondence: $(\overline{\mathfrak{g}}, \sigma, \tau,\langle\rangle) \rightarrow(M, N)$ also keeps each equivalence and translates the direct sum of effective COQ's into the direct product of substantial totally geodesic symmetric submanifolds.

THEOREM 3.3. These correspondences: $(M, N) \rightarrow(\overline{\mathfrak{g}}, \sigma, \tau,\langle>),(\overline{\mathfrak{g}}, \sigma, \tau,\langle>) \rightarrow(M, N)$ are the inverses of each other. 
Proof. Let $(M, N)$ be a pair of compact simply connected riemannian symmetric space $M$ and substantial totally geodesic symmetric submanifold $N$, and $(\bar{g}, \sigma, \tau,\langle\rangle)$ the effective COQ associated with $(M, N)$. Moreover let $\left(M^{\prime}, N^{\prime}\right)$ be the pair associated with the COQ. We show that $\left(M^{\prime}, N^{\prime}\right)$ is equivalent to $(M, N)$. We retain the same notation as above for the correspondence: $(M, N) \rightarrow(\overline{\mathfrak{g}}, \sigma, \tau,\langle>)$ and attach ' to the previous notations for the correspondence: $\left(\overline{\mathfrak{g}}, \sigma, \tau,\langle>) \rightarrow\left(M^{\prime}, N^{\prime}\right)\right.$. Since $\bar{G}^{\prime}, I^{0}(M)$ have the same Lie algebra $\bar{g}$, there exists a Lie group homomorphism $\Phi$ of $\bar{G}^{\prime}$ onto $I^{0}(M)$ whose differential is the identity of $\overline{\mathrm{g}}$. Obviously it holds $\Phi\left(\bar{K}^{\prime}\right) \subset \bar{K}$. Hence $\Phi$ induces a $\bar{G}^{\prime}$-equivariant isometric immersion $\psi$ of $M^{\prime}$ onto $M$ such that $\psi\left(o^{\prime}\right)=p, \psi_{* o^{\prime}}=1_{\bar{p}}$. Here $T_{o^{\prime}} M^{\prime}, T_{p} M$ are identified with the subspace $\bar{p}$ canonically. Since $\psi$ is a covering map and $M$ is simply connected, $\psi$ is an isometry of $M^{\prime}$ onto $M$. Moreover since $T_{o} N^{\prime}, T_{p} N$ are both identified with the subspace $\overline{\mathfrak{p}}_{-}$in $\overline{\mathfrak{p}}$, it follows that $\psi\left(N^{\prime}\right)=N$. Then $\psi$ induces an isometry of $N^{\prime}$ onto $N$ by Lemma 1.2 , (2). Hence $\left(M^{\prime}, N^{\prime}\right)$ is equivalent to $(M, N)$.

Conversely let $(\overline{\mathfrak{g}}, \sigma, \tau,\langle\rangle)$ be an effective COQ and $(M, N)$ the pair associated with the COQ. Moreover let $\left(\bar{g}^{\prime}, \sigma^{\prime}, \tau^{\prime},\left\langle>^{\prime}\right)\right.$ be the effective COQ associated with $(M, N)$. We show that $\left(\overline{\mathfrak{g}}, \sigma, \tau,\langle>)\right.$ is equivalent to $\left(\overline{\mathfrak{g}}^{\prime}, \sigma^{\prime}, \tau^{\prime},\langle\rangle^{\prime}\right)$. We retain the same notations as above for the construction: $(\overline{\mathfrak{g}}, \sigma, \tau,\langle\rangle) \rightarrow(M, N)$ and attach ' to the previous notations for the construction: $(M, N) \rightarrow\left(\overline{\mathfrak{g}}^{\prime}, \sigma^{\prime}, \tau^{\prime},\left\langle>^{\prime}\right)\right.$. Now since $(\overline{\mathfrak{g}}, \sigma)$ is effective, the Lie algebras $\overline{\mathfrak{g}}, \overline{\mathfrak{g}}^{\prime}$ are isomorphic to each other. The isomorphism $\phi$ of $\overline{\mathfrak{g}}$ onto $\overline{\mathfrak{g}}^{\prime}$ is given as follows. For $X \in \bar{g}$, denote by $X^{\prime}$ the Killing vector field on $M^{\prime}$ given by the one parameter subgroup exp $t X$ of $\bar{G}$. Then $\phi$ is defined by $\phi(X)=X^{\prime}$. This $\phi$ satisfies that $\phi(\overline{\mathfrak{f}})=\overline{\mathfrak{f}}^{\prime}, \phi(\overline{\mathfrak{p}})$ $=\overline{\mathfrak{p}}^{\prime}$ and thus it follows $\phi \circ \sigma=\sigma^{\prime} \circ \phi$. (See Helgason [6], p. 243 for these.) Moreover note that $\langle\phi(X), \phi(Y)\rangle_{\overline{\mathfrak{p}}^{\prime}}^{\prime}=\langle X, Y\rangle_{\bar{p}}$ for $X, Y \in \overline{\mathfrak{p}}$ from the definition of \langle\rangle$_{\overline{\mathfrak{p}}^{\prime}}^{\prime}$. Since \langle\rangle$^{\prime},\langle\rangle$ are unique extensions of \langle\rangle$_{\bar{p}^{\prime}}^{\prime},\langle\rangle_{\bar{p}}$ to $\overline{\mathfrak{g}}^{\prime}, \overline{\mathfrak{g}}$, it follows $\langle\phi(X), \phi(Y)\rangle^{\prime}=\langle X, Y\rangle$ for $X$, $Y \in \overline{\mathrm{g}}$. Lastly we show that $\phi \circ \tau=\tau^{\prime} \circ \phi$. Since $\phi \circ \tau, \tau^{\prime} \circ \phi$ are Lie algebra isomorphisms and $\overline{\mathfrak{f}}=[\overline{\mathfrak{p}}, \overline{\mathfrak{p}}]$, we may show the equality on $\overline{\mathfrak{p}}$. Let $X \in \overline{\mathfrak{p}}_{-}$. Then $(\exp t X)(o)=$ $(\exp t \phi(X))(o)$ is a geodesic in $N$. Hence it follows

$$
\begin{aligned}
\left(\exp t \tau^{\prime}(\phi(X))\right)(o) & =\tau^{\prime}(\exp t \phi(X))(o)=t_{o}((\exp t \phi(X))(o)) \\
& =(\exp -t \phi(X))(o) .
\end{aligned}
$$

Since $\phi(X), \tau^{\prime}(\phi(X)) \in \bar{p}^{\prime}$, it holds exp $t \tau^{\prime}(\phi(X))=\exp -t \phi(X)$ and thus $\tau^{\prime}(\phi(X))=-\phi(X)$. Similarly we have $\tau^{\prime}(\phi(X))=\phi(X)$ for $X \in \overline{\mathfrak{p}}_{+}$. These imply that $\tau^{\prime} \circ \phi=\phi \circ \tau$ on $\bar{p}$ and thus on $\overline{\mathfrak{g}}$. By these arguments $\left(\overline{\mathfrak{g}}^{\prime}, \sigma^{\prime}, \tau^{\prime},\langle\rangle^{\prime}\right)$ is equivalent to $(\overline{\mathfrak{g}}, \sigma, \tau,\langle\rangle)$. $\quad$ q. e. $\mathrm{d}$.

REMARK 3.4. Let $(\overline{\mathfrak{g}}, \sigma, \tau,\langle>)$ be an effective COQ. Then $(\overline{\mathfrak{g}}, \tau, \sigma,\langle>)$ is also an effective COQ. Let $(M, N),\left(M^{*}, N^{*}\right)$ be the pairs associated with $(\overline{\mathfrak{g}}, \sigma, \tau,\langle>),(\overline{\mathfrak{g}}, \tau, \sigma$, $\left\langle>\right.$ ) respectively. Then $N$ is locally isometric to $N^{*}$ as riemannian manifold. Also fix $p \in N$ and let $N^{\perp} \subset M$ be the totally geodesic symmetric submanifold such that $N^{\perp} \ni p$, $T_{p} N^{\perp}=N_{p} N$. Then the COQ associated with $\left(M, N^{\perp}\right)$ is isomorphic to $(\overline{\mathrm{g}}, \sigma, \sigma \circ \tau,\langle>)$. 
This COQ is not necessarily effective since $(\overline{\mathfrak{g}}, \sigma \circ \tau)$ is not necessarily effective.

REMARK 3.5. Totally geodesic symmetric submanifolds of simply connected riemannian symmetric spaces of noncompact type, can be got through the duality between symmetric spaces of compact type and those of noncompact type.

Now consider triples $(\overline{\mathfrak{g}}, \sigma, \tau)$ underlying COQ's. That is, $\bar{g}$ is a Lie algebra of compact type and $\sigma, \tau$ are involutive automorphisms of $\overline{\mathfrak{g}}$ such that $[\sigma, \tau]=0$. This triple $(\overline{\mathfrak{g}}, \sigma, \tau)$ is called a compact triple. If the symmetric Lie algebras $(\overline{\mathfrak{g}}, \sigma),(\overline{\mathfrak{g}}, \tau)$ are effective, it is also called effective. Among effective compact triples we can define the "equivalence" and the "direct sum" canonically. On the other hand a symmetric Lie algebra $(\hat{\mathfrak{g}}, \hat{\tau})$ is called of noncompact type if the underlying Lie algebra $\hat{g}$ is a semisimple Lie algebra of noncompact type. Among effective symmetric Lie algebras of noncompact type we can also define the "equivalence" and the "direct sum" canonically. Here we see that there exists a one-to-one correspondence between these two kinds of objects.

Let $(\overline{\mathfrak{g}}, \sigma, \tau)$ be an effective compact triple and let $\overline{\mathfrak{g}}=\overline{\mathfrak{f}} \oplus \overline{\mathfrak{p}}$ be the eigenspace decomposition by $\sigma$ into the $(+1)$-eigenspace $\overline{\mathfrak{f}}$ and the $(-1)$-eigenspace $\overline{\mathfrak{p}}$. Then $\overline{\mathfrak{g}}=\overline{\mathfrak{f}}+\sqrt{-1} \overline{\mathfrak{p}}$ has the structure of Lie algebra of noncompact type canonically. Define an involutive automorphism $\hat{\tau}$ of $\hat{\mathfrak{g}}$ by $\hat{\tau}(T+\sqrt{-1} X)=\hat{\tau}(T)+\sqrt{-1} \hat{\tau}(X)$ for $T \in \overline{\mathfrak{t}}, X \in \overline{\mathfrak{p}}$. Then, since $(\overline{\mathfrak{g}}, \tau)$ is effective, $(\hat{\mathfrak{g}}, \hat{\tau})$ is also effective. Hence $(\hat{\mathfrak{g}}, \hat{\tau})$ is an effective symmetric Lie algebra of noncompact type.

Conversely let $(\hat{\mathfrak{g}}, \hat{\tau})$ be an effective symmetric Lie algebra of noncompact type. Take a Cartan involution $\hat{\sigma}$ of $\hat{\boldsymbol{g}}$ such that $[\hat{\boldsymbol{\sigma}}, \hat{\tau}]=0$. Such $\hat{\sigma}$ always exists and is unique in the sense. If $\hat{\sigma}, \hat{\sigma}^{\prime}$ are such Cartan involutions, there exists a Lie algebra isomorphism $\hat{\phi}$ of $\hat{g}$ such that $\hat{\phi} \circ \hat{\sigma}=\hat{\sigma}^{\prime} \circ \hat{\phi},[\hat{\phi}, \hat{\tau}]=0$. (cf. See the arguement in Helgason [6], p. 184 for the uniqueness.) Let $\hat{\mathbf{g}}=\overline{\mathfrak{f}} \oplus \overline{\mathfrak{p}}$ be the eigenspace decomposition by $\hat{\sigma}$ into the $(+1)$-eigenspace $\overline{\mathfrak{f}}$ and the $(-1)$-eigenspace $\overline{\mathfrak{p}}$. Then $\overline{\mathfrak{g}}=\overline{\mathfrak{f}} \oplus \sqrt{-1} \overline{\mathfrak{p}}$ has the structure of Lie algebra of compact type. Define involutive automorphisms $\sigma, \tau$ of $\overline{\mathrm{g}}$ by $\sigma(T+\sqrt{-1} X)=T-\sqrt{-1} X$, $\tau(T+\sqrt{-1} X)=\hat{\tau}(T)+\sqrt{-1} \hat{\tau}(X)$ for $T \in \overline{\mathfrak{f}}, X \in \overline{\mathfrak{p}}$. Obviously the symmetric Lie algebras $(\overline{\mathfrak{g}}, \sigma),(\overline{\mathfrak{g}}, \tau)$ are effective. Hence the triple $(\overline{\mathfrak{g}}, \sigma, \tau)$ is an effective compact triple.

Proposition 3.6. Our correspondences: $(\overline{\mathfrak{g}}, \sigma, \tau) \rightarrow(\hat{\mathfrak{g}}, \hat{\tau}), \quad(\hat{\mathfrak{g}}, \hat{\tau}) \rightarrow(\overline{\mathfrak{g}}, \sigma, \tau)$ keep each equivalence and each direct sum. Moreover they are the inverse of each other.

REMARK 3.7. An object is called irreducible if it is not decomposed into any proper direct sums. Irreducible and effective symmetric Lie algebras have been classified in Berger [2]. Moreover an effective $\mathrm{COQ}$ is irreducible if and only if the underlying effective compact triple is irreducible. 


\section{Substantial symmetric submanifolds}

Let $(\overline{\mathfrak{g}}, \sigma, \tau,\langle\rangle)$ be an effective COQ and $\overline{\mathfrak{g}}=\overline{\mathfrak{f}} \oplus \overline{\mathfrak{p}}$ the eigenspace decomposition by $\sigma$. Moreover let $\overline{\mathfrak{f}}=\overline{\mathfrak{f}}_{+} \oplus \overline{\mathfrak{f}}_{-}, \overline{\mathfrak{p}}=\overline{\mathfrak{p}}_{+} \oplus \overline{\mathfrak{p}}_{-}$be eigenspace decompositions by the restrictions of $\tau$ to $\overline{\mathfrak{f}}, \overline{\mathfrak{p}}$ into $(+1)$-eigenspaces $\overline{\mathfrak{f}}_{+}, \overline{\mathfrak{p}}_{+}$and $(-1)$-eigenspaces $\overline{\mathfrak{f}}_{-}, \overline{\mathfrak{p}}_{-}$respectively. Put $\overline{\mathfrak{f}}^{*}=\overline{\mathfrak{f}}_{+} \oplus \overline{\mathfrak{p}}_{+}$and $\overline{\mathfrak{p}}^{*}=\overline{\mathfrak{f}}_{-} \oplus \overline{\mathfrak{p}}_{-}$. Then $\overline{\mathfrak{g}}=\overline{\mathfrak{f}}^{*} \oplus \overline{\mathfrak{p}}^{*}$ is the eigenspace decomposition by $\tau$. A linear subspace $\mathfrak{m}$ in $\overline{\mathfrak{p}}^{*}$ is called a Lie triple system if it holds $[[\mathfrak{m}, \mathfrak{m}], \mathfrak{m}] \subset \mathfrak{m}$. Consider a Lie triple system $\mathfrak{m} \subset \overline{\mathfrak{p}}^{*}$ such that

(L.1) $[\mathfrak{m}, \mathfrak{m}] \subset \overline{\mathfrak{f}}_{+}$

and

(L.2) the orthogonal projection: $\mathfrak{m} \rightarrow \overline{\mathfrak{p}}_{-}$is a linear isomorphism. And denote by $(\overline{\mathfrak{g}}, \sigma$, $\tau,\langle>: \mathfrak{m})$ the pair of effective COQ $(\overline{\mathfrak{g}}, \sigma, \tau,\langle>)$ and such Lie triple system $\mathfrak{m}$. We call this pair a $Q L$-pair. Two QL-pairs $\left(\overline{\mathfrak{g}}, \sigma, \tau,\langle>: \mathfrak{m}),\left(\overline{\mathfrak{g}}^{\prime}, \sigma^{\prime}, \tau^{\prime},\langle\rangle^{\prime}: \mathfrak{m}^{\prime}\right)\right.$ is said to be equivalent to each other if there exists an isomorphism $\phi$ of the $\operatorname{COQ}(\overline{\mathfrak{g}}, \sigma, \tau,\langle>)$ onto the $\operatorname{COQ}\left(\bar{g}^{\prime}, \sigma^{\prime}, \tau^{\prime},\langle>\rangle^{\prime}\right)$ such that $\phi(\mathfrak{m})=\mathfrak{m}^{\prime}$. Moreover the "direct sum" of finite QL-pairs is defined canonically.

Let $(M, S)$ be a pair of compact simply connected riemannian symmetric space $M$ and substantial symmetric submanifold $S$ of $M$. Moreover fix $p \in S$ and let $N$ be the associated substantial totally geodesic symmetric submanifold through $p$, with an effective COQ $(\overline{\mathfrak{g}}$, $\sigma, \tau,\left\langle>\right.$ ). Let $\mathfrak{m}$ be the subspace in $\overline{\mathfrak{p}}^{*}$, of Killing vector fields on $M$ whose restrictions to $S$ are also Killing vector fields on $S$.

LEMMA 4.1. The pair $(\overline{\mathfrak{g}}, \sigma, \tau,\langle>$ : m) is a QL-pair.

ProOF. Obviously $\mathfrak{m}$ is a Lie triple system since $\overline{\mathfrak{p}}^{*}$ is so.

Recall that the projection $\pi$ is defined by the relation: $\pi(\bar{X})=X$ for Killing vector fields $\bar{X}$ on $M$ projectable to Killing vector fields $X$ on $S$. Moreover recall that the vector space $p$ is defined as the (-1)-eigenspace of $\delta$. Here $\delta$ is the involutive automorphism of $g$ defined from $s_{p}$. Then it holds $\pi(\mathfrak{m}) \subset \mathfrak{p}$ since $\delta \circ \pi=\pi \circ \tau$ on $\mathfrak{m}$.

Firstly we show that the projection $\pi$ is a linear isomorphism of $\mathfrak{m}$ onto $\mathfrak{p}$. Let $X \in \mathfrak{p}$ and take the integral complete geodesic $\gamma(t)$ such that $\gamma(0)=p$. Consider the Killing vector fields $\bar{X}$ on $M$ which generates the one parameter subgroup $T(t)$. Since $\left(t_{p} \circ T(t) \circ t_{p}\right)(p)$ $=T(-t)(p)=\gamma(-t)$ and $\left(t_{p} \circ T(t) \circ t_{p}\right)_{*_{p}}=(T(-t))_{*_{p}}$ by (2.3), (2.5), (2.7), it follows $t_{p} \circ T(t) \circ t_{p}=T(-t)$ and thus $\tau(\bar{X})=-\bar{X}$. Moreover since $\pi(\bar{X})=X$, it follows $\bar{X} \in \mathfrak{m}$. Hence $\pi$ is surjective. Next let $\bar{X} \in \mathfrak{m}$ such that $\pi(\bar{X})=0$. Then it follows $(\exp t \bar{X})(p)=p$ and $(\exp t \bar{X})_{*} T_{p} S=T_{p} S,(\exp t \bar{X})_{*} N_{p} S=N_{p} S$. Moreover since $\tau(\bar{X})=-\bar{X}$, it follows $t_{p} \circ \exp t \bar{X} \circ t_{p}=\exp -t \bar{X}$. Hence it holds $(\exp t \bar{X})_{*_{p}}=(\exp -t \bar{X})_{*_{p}}$. Since these are both isometries, it follows $\exp t \bar{X}=\exp -t \bar{X}$ and thus $\bar{X}=0$. This implies that $\pi$ is injective. Hence $\pi$ is a linear isomorphism of $\mathfrak{m}$ onto $\mathfrak{p}$. 
Now we show that $\mathfrak{m}$ satisfies the conditions (L.1), (L.2). Let $\bar{X}, \bar{Y} \in \mathfrak{m}$. Then it holds $\pi([\bar{X}, \bar{Y}]) \in \mathfrak{f}$ and thus $[\bar{X}, \bar{Y}] \in \overline{\mathfrak{f}}$, where $\mathfrak{f}$ denotes the $(+1)$-eigenspace of $\delta$. Moreover since $\tau([\bar{X}, \bar{Y}])=[\bar{X}, \bar{Y}]$, it follows $[\bar{X}, \bar{Y}] \in \overline{\mathfrak{f}}_{+}$. Hence $\mathfrak{m}$ satisfies the condition (L.1). Next let $\bar{X} \in \mathfrak{m} \cap \overline{\mathfrak{f}}_{-}$. Then it follows $\pi(\bar{X}) \in \mathfrak{t} \cap \mathfrak{p}$ and thus $\pi(\bar{X})=0$. Since $\pi$ is injective, it holds $\bar{X}=0$. This implies that the projection: $\mathfrak{m} \rightarrow \overline{\mathfrak{p}}_{-}$is injective. Since $\operatorname{dim} \mathfrak{m}=\operatorname{dim} \mathfrak{p}=\operatorname{dim} \overline{\mathfrak{p}}_{-}$, it is also surjective. Hence $m$ satisfies the condition (L.2).

q. e. d.

REMARK 4.2. The Lie triple system $m$ consists of all Killing vector fields on $M$ which generate the one parameter subgroups $T(t)$.

Conversely let $(\overline{\mathfrak{g}}, \sigma, \tau,\langle>: \mathfrak{m})$ be a QL-pair and $(M, N)$ the pair associated with the effective $\operatorname{COQ}(\overline{\mathfrak{g}}, \sigma, \tau,\langle\rangle)$. Put $\mathfrak{h}_{\mathfrak{m}}=[\mathfrak{m}, \mathfrak{m}]$ and $\mathfrak{g}_{\mathfrak{m}}=\mathfrak{h}_{\mathfrak{m}} \oplus \mathfrak{m}$. Since $\mathfrak{m}$ is a Lie triple system, $\mathfrak{g}_{\mathfrak{m}}$ is a Lie subalgebra of $\overline{\mathfrak{g}}$. Let $G_{\mathfrak{m}}$ be the connected Lie subgroup of $I^{0}(M)$ with Lie algebra $g_{\mathfrak{m}}$ and $S$ the orbit of the base point $o$ by $G_{\mathfrak{m}}$.

LEMMA 4.3. The submanifold $S$ is a substantial symmetric submanifold with $N$ as associated totally geodesic symmetric submanifold.

Proof. Obviously $S$ is tangent to $N$ at $o$. We show that $S$ is symmetric. Since $S$ is $G_{\mathrm{m}}$ equivariant, we may show the existence of the extrinsic, the intrinsic symmetries $t_{o}, s_{o}$ at $o$. Now consider the involutive automorphism $\tau$ of $\bar{g}$ and denote by the same notation the Lie group automorphism of $\bar{G}$ whose differential is $\tau$. Since $\tau\left(g_{\mathfrak{m}}\right)=g_{\mathfrak{m}}, \tau$ induces a Lie group automorphism $\delta$ of $G_{\mathrm{m}}$. Let $t_{o}, s_{o}$ be the smooth mappings of $M, S$ induced from $\tau, \delta$ respectively. Then $s_{o}$ is the restriction of $t_{o}$ to $S$. Since $\tau$ leaves \langle\rangle$_{\mathfrak{p}}$ invariant and satisfies $\tau(X)$ $=-X, \tau(\xi)=\xi$ for $X \in \overline{\mathfrak{p}}_{-}, \xi \in \overline{\mathfrak{p}}_{+}$, the mapping $t_{o}$ is the extrinsic symmetry at $o$ and thus $s_{o}$ is the intrinsic symmetry at $o$. Hence $S$ is symmetric.

It is obvious by Lemma 2.12 that $S$ is substantial.

q.e.d.

Now our correspondences: $(M, S) \rightarrow(\overline{\mathfrak{g}}, \sigma, \tau,\langle>: \mathfrak{m}),(\overline{\mathfrak{g}}, \sigma, \tau,\langle>: \mathfrak{m}) \rightarrow(M, S)$ keep each equivalence and moreover the followings hold.

THEOREM 4.4. These correspondences are the inverses of each other and translate the direct product of substantial symmetric submanifolds into the direct sum of QL-pairs.

Proof. Let $(M, S)$ be a pair of compact simply connected riemannian symmetric space $M$ and substantial symmetric submanifold $S$ of $M$, and $(\overline{\mathfrak{g}}, \sigma, \tau,\langle>: \mathfrak{m})$ the QL-pair associated with $(M, S)$. Moreover let $\left(M^{\prime}, S^{\prime}\right)$ be the pair associated with the QL-pair. We show that $\left(M^{\prime}, S^{\prime}\right)$ is equivalent to $(M, S)$. We retain the same notations as in the proof of Theorem 3.3. Now firstly we show that $\pi$ is a Lie algebra isomorphism of $g_{\mathfrak{m}}$ onto $g_{0}$. Obviously it holds $\pi\left(g_{\mathfrak{m}}\right)=g_{0}$. Let $X \in g_{\mathfrak{m}}$ such that $\pi(X)=0$. Since $\pi$ is a linear isomorphism of $\mathfrak{m}$ onto $\mathfrak{p}$, it follows $X \in \mathfrak{h}_{\mathfrak{m}}$ and $\operatorname{ad}_{\mathfrak{m}}(X)(Y)=0$ for $Y \in \mathfrak{m}$. Note that the adjoint representation $\operatorname{ad}_{\mathfrak{m}}$ of $\mathfrak{h}_{\mathfrak{m}}$ to $\mathfrak{m}$ is faithful since $\mathfrak{g}_{\mathfrak{m}}$ admits a positive definite inner product such that 
$\operatorname{ad}_{\mathfrak{g}_{\mathfrak{m}}}(Y), Y \in \mathfrak{g}_{\mathfrak{m}}$, are skew symmetric. Hence it holds $X=0$. This implies that $\pi$ is injective and thus is a Lie algebra isomorphism of $g_{\mathfrak{m}}$ onto $g_{0}$. Let $\imath$ be the inclusion of $S$ into $M$. Then $\pi^{-1}$ is an $l_{*}$-related Lie homomorphism of $g_{0}$ into $g_{\mathfrak{m}} \subset \overline{\mathfrak{g}}$. Denote by $G_{\mathfrak{m}}$ the connected Lie subgroup of $I^{0}(M)$ with Lie algebra $g_{\mathfrak{m}}$. By Cororally 2.8 the group $G_{\mathfrak{m}}$ is a Lie transformation group acting on $S$ transitively. Here recall the Lie group homomorphism $\Phi$ of $\bar{G}^{\prime}$ onto $I^{0}(M)$, which induces the isometry $\psi$ of $M^{\prime}$ onto $M$. Then $\Phi$ induces a Lie group homomorphism of $G_{\mathrm{m}}^{\prime}$ to $G_{\mathrm{m}}$ and thus a smooth mapping of $S^{\prime}$ to $S$. Obviously it is the restriction of $\psi$ to $S^{\prime}$ and thus an isometry of $S^{\prime}$ onto $S$. This implies that $\left(M^{\prime}, S^{\prime}\right)$ is equivalent to $(M, S)$.

Next let $(\overline{\mathfrak{g}}, \sigma, \tau,\langle>: \mathfrak{m})$ be a QL-pair and $(M, S)$ the pair associated with the QL-pair. Moreover let $\left(\overline{\mathfrak{g}}^{\prime}, \boldsymbol{\sigma}^{\prime}, \tau^{\prime},\langle\rangle^{\prime}: \mathfrak{m}^{\prime}\right)$ be the QL-pair associated with $(M, S)$. We show that $\left(\overline{\mathfrak{g}}^{\prime}, \sigma^{\prime}, \tau^{\prime},\left\langle>^{\prime}: \mathfrak{m}^{\prime}\right)\right.$ is equivalent to $(\overline{\mathfrak{g}}, \sigma, \tau,\langle>: \mathfrak{m})$. Again we retain the same notations as in the proof of Theorem 3.3. Recall the isomorphism $\phi$ of the COQ $(\overline{\mathfrak{g}}, \sigma, \tau,\langle\rangle)$ onto the COQ $\left(\overline{\mathfrak{g}}^{\prime}, \sigma^{\prime}, \tau^{\prime},\langle\rangle^{\prime}\right)$. Obviously the restrictions of $\phi(X), X \in \mathfrak{m}$, to $S^{\prime}$ are Killing vector fields on $S^{\prime}$. Moreover since $\phi \circ \tau=\tau^{\prime} \circ \phi$, it follows $\tau^{\prime}(\phi(X))=-\phi(X)$ for $X \in \mathfrak{m}$. Hence it holds $\phi(\mathfrak{m}) \subset \mathfrak{m}^{\prime}$. Since $\operatorname{dim} \mathfrak{m}=\operatorname{dim} \mathfrak{m}^{\prime}$, it follows $\phi(\mathfrak{m})=\mathfrak{m}^{\prime}$. This implies that $(\overline{\mathfrak{g}}, \sigma, \tau,\langle>: \mathfrak{m}$ ) is equivalent to $\left(\bar{g}^{\prime}, \sigma^{\prime}, \tau^{\prime},\left\langle>^{\prime}: \mathfrak{m}^{\prime}\right)\right.$.

The second claim is obvious.

q.e.d.

REMARK 4.5. Let $(\overline{\mathfrak{g}}, \sigma, \tau,\langle>: \mathfrak{m})$ be a QL-pair and $(M, S)$ the pair associated with the QL-pair. Moreover let $M^{*}$ be the compact simply connected riemannian symmetric space defined by the orthogonal symmetric Lie algebra $(\overline{\mathfrak{g}}, \tau,\langle\rangle)$. Then, since $\mathfrak{m}$ is a Lie triple system in $\overline{\mathfrak{p}}^{*}$, it is identified with a curvature-invariant subspace in the tangent space $T_{o^{*}} M^{*}$ at the base point $o^{*}$. Hence it defines a unique complete totally geodesic submanifold $S^{*}$ of $M^{*}$ such that $S^{*} \ni o^{*}, T_{o^{*}} S^{*}=\mathrm{m}$. The study of QL-pairs is to see $S^{*}$ satisfying the conditions (L.1), (L.2). Generally many examples of totally geodesic submanifolds in riemannian symmetric spaces are known in Nagano-Chen [3]. Lastly note that $S, S^{*}$ are locally isomorphic as symmetric space but they are not always isometric even locally.

Now let $(\overline{\mathfrak{g}}, \sigma, \tau,\langle>: \mathfrak{m})$ be a QL-pair. By the condition (L.2) there exists a unique linear map $\lambda$ of $\overline{\mathfrak{p}}_{-}$to $\overline{\mathfrak{f}}_{-}$such that

$$
\mathfrak{m}=\left\{X+\lambda(X) \in \overline{\mathfrak{p}}_{-} \oplus \overline{\mathfrak{f}}_{-} ; X \in \overline{\mathfrak{p}}_{-}\right\} .
$$

Then the condition (L.1) and the condition that $m$ is a Lie triple system, can be rewritten as follows respectively.

$$
[X, \lambda(Y)]=[Y, \lambda(X)]
$$

$$
\lambda([X, Y], Z])+\lambda([[\lambda(X), \lambda(Y)], Z])=[[X, Y], \lambda(Z)]+[[\lambda(X), \lambda(Y)], \lambda(Z)]
$$

for $X, Y, Z \in \overline{\mathfrak{p}}_{-}$. We call this pair $(\overline{\mathfrak{g}}, \sigma, \tau,\langle>: \lambda)$ a $Q \Lambda$-pair. Two $Q \Lambda$-pairs $(\overline{\mathfrak{g}}, \sigma, \tau,\langle>$ : $\lambda),\left(\overline{\mathfrak{g}}^{\prime}, \sigma^{\prime}, \tau^{\prime},\langle\rangle^{\prime}: \lambda^{\prime}\right)$ are equivalent to each other if there exists an isomorphism $\phi$ of $(\overline{\mathrm{g}}$, $\sigma, \tau,\left\langle>\right.$ ) onto $\left(\bar{g}^{\prime}, \sigma^{\prime}, \tau^{\prime},\langle\rangle^{\prime}\right)$ such that $\phi \circ \lambda=\lambda^{\prime} \circ \phi$ on $\bar{p}_{-}$. Moreover define the "direct 
sum" of finite $Q \Lambda$-pairs canonically. Obviously we have the following.

CORORALLY 4.6. There exists a one-to-one correspondence between the equivalent classes of substantial symmetric submanifold and those of $Q \Lambda$-pair, and the correspondence translates the direct product into the direct sum.

\section{Substantial symmetric submanifolds with specific effective $\mathrm{COQ}$}

Let $(\bar{g}, \sigma, \tau,\langle>)$ be an effective COQ and $(M, N)$ be the pair associated with the COQ. Note that $\overline{\mathfrak{p}}_{-}$is a Lie triple system and consider the adjoint representation $\operatorname{ad}_{\overline{\mathfrak{p}}_{-}}$of $\overline{\mathfrak{h}}_{-}$to $\overline{\mathfrak{p}}_{-}$, where $\overline{\mathfrak{h}}_{-}=\left[\overline{\mathfrak{p}}_{-}, \overline{\mathfrak{p}}_{-}\right]$. Since the representation is faithful, the Lie algebra $\overline{\mathfrak{h}}_{-}$is isomorphic to the holonomy algebra of $N$.

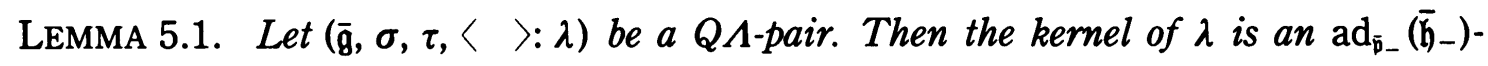
module in $\overline{\mathrm{p}}_{-}$.

Proof. Let $X, Y, Z \in \bar{p}_{-}$. We may show that $\lambda([[X, Y], Z])=0$ if $\lambda(Z)=0$. Assume that $\lambda(Z)=0$. By the condition ( 1.2$)$ it follows

$$
\lambda([[X, Y], Z])+\lambda([[\lambda(X), \lambda(Y)], Z])=0
$$

and moreover, by the condition ( $\Lambda .1)$, it follows

$$
\begin{aligned}
{[[\lambda(X), \lambda(Y)], Z] } & =-[[\lambda(Y), Z], \lambda(X)]-[[Z, \lambda(X)], \lambda(Y)] \\
& =-[[\lambda(Z), Y], \lambda(X)]-[[X, \lambda(Z)], \lambda(Y)]=0 .
\end{aligned}
$$

Hence we have $\lambda([[X, Y], Z])=0$.

q.e.d.

THEOREM 5.2. Let $(\overline{\mathfrak{g}}, \sigma, \tau,\langle>)$ be an effective COQ such that

(1) $\overline{\mathfrak{p}}_{-}$is irreducible as $\mathrm{ad}_{\overline{\mathfrak{p}}_{-}}\left(\overline{\mathfrak{h}}_{-}\right)$-module and

(2) $\operatorname{dim} \overline{\mathfrak{t}}_{-}<\operatorname{dim} \overline{\mathfrak{p}}_{-}$.

Let $(M, N)$ be the pair associated with the COQ and $S$ a substantial symmetric submanifold with $N$ as the associated totally geodesic symmetric submanifold. Then $(M, S)$ is equivalent to $(M, N)$ and particulary $S$ is totally geodesic.

Proof. Let $(\overline{\mathfrak{g}}, \sigma, \tau,\langle>: \lambda)$ be the $Q \Lambda$-pair associated with $(M, S)$. Since the kernel of $\lambda$ is an $\operatorname{ad}_{\overline{\mathfrak{p}}_{-}}\left(\overline{\mathfrak{h}}_{-}\right)$-module, it is $\{0\}$ or $\overline{\mathfrak{p}}_{-}$by the condition (1). Moreover since $\lambda$ is not injective by the condition (2), the kernal is $\bar{p}_{-}$and thus it follows $\lambda=0$. This implies that $(M, S)$ is equivalent to $(M, N)$.

REMARK 5.3. Let $\left(M^{*}, N^{*}\right)$ be the pair associated with the COQ $(\overline{\mathrm{g}}, \tau, \sigma,\langle>)$ and $N^{* \perp}$ the totally geodesic symmetric submanifold perpendicular to $N^{*}$ at the base point $o^{*}$. Then it holds $\operatorname{dim} \overline{\mathfrak{t}}_{-}=\operatorname{dim} N^{* \perp}, \operatorname{dim} \overline{\mathfrak{p}}_{-}=\operatorname{dim} N=\operatorname{dim} N^{*}$. 
Now among the irreducible effective COQ's there exist many examples satisfying the conditions (1), (2). We introduce one of them. Let $M$ be the $n(\geqq 2)$-dimensional complex quadric $C Q_{n}$ and $N$ the $n$-dimensional sphere $S^{n}$ imbedded in $C Q_{n}$ as totally real totally geodesic symmetric submanifold. Then $\left(M^{*}, N^{*}\right)$ is equivalent to $\left(S^{n+1}, S^{n}\right)$. Hence the $\operatorname{COQ}(\overline{\mathfrak{g}}, \sigma, \tau,\langle\rangle)$ associated with $(M, N)$ satisfies the conditions (1), (2). (This pair $\left(C Q_{n}, S^{n}\right)$ appears as $\left(M^{*}, N^{*}\right)$ in Table II, No. $13, i=1$.)

REMARK 5.4. Assume that $M$ has rank one. Then $(M, N)$ is equivalent to one of the followings: $\left(S^{n}, S^{r}\right),\left(C P_{n}, C P_{r}\right),\left(C P_{n}, \boldsymbol{R P} P_{n}\right),\left(H P_{n}, H P_{r}\right),\left(H P_{n}, C P_{n}\right),\left(C a y P_{2}, S^{8}\right),(\boldsymbol{C a y}$ $\left.P_{2}, \boldsymbol{H} P_{2}\right)$. Here $\boldsymbol{R} P_{n}, \boldsymbol{C P} P_{n}, \boldsymbol{H} P_{n}, \boldsymbol{C a y} P_{2}$ denote the projective $n$-spaces over fields $\boldsymbol{R}, \boldsymbol{C}, \boldsymbol{H}$ respectively and the Cayley projective plane, and the inclusions: $N \rightarrow M$ are standard. Now symmetric submanifolds $S$, with these $N$ as associated totally geodesic symmetric submanifold, have been all classified and among them there exist many examples which are not totally geodesic. (For the classifications see [17], [18], [14], [21], [22].) Assume that $(M, N)$ has a symmetric submanifold which is not totally geodesic. Then, since $N$ is irreducible, it holds $\operatorname{dim} \overline{\mathfrak{f}}_{-} \geqq \operatorname{dim} \overline{\mathfrak{p}}_{-}$by Theorem 5.2 .

Now we consider a series of effective COQ's satisfying $\operatorname{dim} \overline{\mathfrak{t}}_{-}=\operatorname{dim} \overline{\mathfrak{p}}_{-}$. They are defined as effective $\operatorname{COQ}(\overline{\mathfrak{g}}, \sigma, \tau,\langle>)$ satisfying the following conditions.

(C.1) There exists some $H_{0} \in \overline{\mathfrak{p}}_{+}$such that $\operatorname{ad}_{\tilde{p}^{*}}\left(H_{0}\right)^{2}=-1_{\bar{p}^{*}}$, where $1_{\overline{\mathfrak{p}}^{*}}$ denotes the identity of $\overline{\mathfrak{p}}^{*}$. Denote $J=\operatorname{ad}_{\tilde{p}^{*}}\left(H_{0}\right)$.

(C.2) The linear space $\overline{\mathfrak{p}}_{+}$is decomposed into the sum of subspaces $\overline{\mathfrak{a}}, \overline{\mathfrak{b}}$ such that

$$
J \circ \operatorname{ad}_{\overline{\mathfrak{p}}^{*}}(T)=\left\{\begin{array}{rll}
-\operatorname{ad}_{\overline{\mathfrak{p}}^{*}}(T) \circ J & \text { if } & T \in \overline{\mathfrak{a}}, \\
\operatorname{ad}_{\overline{\mathfrak{p}}}(T) \circ J & \text { if } & T \in \overline{\mathfrak{b}} .
\end{array}\right.
$$

Obviously it holds $\operatorname{dim} \overline{\mathfrak{t}}_{-}=\operatorname{dim} \overline{\mathfrak{p}}_{-}$.

Generally let $(\overline{\mathfrak{g}}, \sigma, \tau,\langle>: \lambda)$ be a $Q \Lambda$-pair. Then it follows

$$
\operatorname{ad}(T) \circ \lambda=-\lambda^{t} \circ \operatorname{ad}(T)
$$

on $\overline{\mathfrak{p}}_{-}$for $T \in \overline{\mathfrak{p}}_{+}$, where $(*)^{t}$ denotes the transposed map of (*). In fact, let $X, Y \in \overline{\mathfrak{p}}_{-}$. By the condition $(\Lambda .1)$ it follows

$$
\begin{aligned}
\langle\operatorname{ad}(T) \circ \lambda(X), Y\rangle & =\langle[\lambda(X), Y], T\rangle=\langle[\lambda(Y), X], T\rangle \\
& =\left\langle-\lambda^{t} \circ \operatorname{ad}(T) X, Y\right\rangle .
\end{aligned}
$$

Hence the equality (5.1) holds.

Next assume that the underlying $\operatorname{COQ}(\overline{\mathfrak{g}}, \sigma, \tau,\langle\rangle)$ satisfies the conditions (C.1), (C.2). Then it follows

$$
\lambda \circ \operatorname{ad}(T)=-\operatorname{ad}(T) \circ \lambda^{t}
$$

on $\overline{\mathfrak{t}}_{-}$for $T \in \overline{\mathfrak{p}}_{+}$. In fact, consider the endomorphism $J \circ \lambda$ of $\overline{\mathfrak{p}}_{-}$. Then it is symmetric by (5.1). Since $J \circ \lambda=-J \circ \lambda \circ J \circ J$ and $(J \circ \lambda)^{t}=-J \circ(\lambda \circ J)^{t} \circ J$, the endomorphism $\lambda \circ J$ of $\overline{\mathfrak{f}}_{-}$is 
also symmetric. Let $T \in \overline{\mathfrak{a}}$. Then it follows

$$
\begin{aligned}
J \circ \operatorname{ad}(T) \circ \lambda^{t} & =-\operatorname{ad}(T) \circ J \circ \lambda^{t}=\operatorname{ad}(T) \circ \lambda \circ J=-\lambda^{t} \circ \operatorname{ad}(T) \circ J \\
& =\lambda^{t} \circ J \circ \operatorname{ad}(T)=-J \circ \lambda \circ \operatorname{ad}(T)
\end{aligned}
$$

by (5.1). Hence the equality (5.2) holds for $T \in \overline{\mathfrak{a}}$. In the same way it also holds for $T \in \overline{\mathfrak{b}}$.

Define an endomorphism $\Lambda_{\lambda}$ of $\overline{\mathfrak{p}}^{*}$ by $\Lambda_{\lambda}(X+\xi)=\lambda(X)-\lambda^{t}(\xi)$ for $X \in \overline{\mathfrak{p}}_{-}, \xi \in \overline{\mathfrak{f}}_{-}$.

LEMMA 5.3. The endomorphism $\Lambda_{\lambda}$ is skew symmetric and it holds $\left[a d_{\bar{p}^{*}}(T), \Lambda_{\lambda}\right]=0$ for $T \in \overline{\mathfrak{h}}_{+} \oplus \overline{\mathfrak{p}}_{+}$, where $\overline{\mathfrak{h}}_{+}=\left[\overline{\mathfrak{p}}_{+}, \overline{\mathfrak{p}}_{+}\right]$.

Proof. The first claim is obvious. For the second claim it holds $\left[\operatorname{ad}_{\tilde{p}^{*}}(T), \Lambda_{\lambda}\right]=0$ for $T \in \overline{\mathfrak{p}}_{+}$from (5.1), (5.2), and consequently for $T \in \overline{\mathfrak{h}}_{+}$. Hence the equality holds for all $T \in \overline{\mathfrak{h}}_{+} \oplus \overline{\mathbf{p}}_{+}$.

q.e.d.

Now we consider the following two kinds of effective COQ's satisfying the conditions (C.1), (C.2). We retain the above notations, i.e., $(M, N),\left(M^{*}, N^{*}\right)$ denote the pairs associated with the effective COQ's $(\overline{\mathfrak{g}}, \sigma, \tau,\langle>),(\overline{\mathfrak{g}}, \tau, \sigma,\langle>)$ respectively.

\section{Totally complex totally geodesic symmetric submanifolds $N^{*}$ of compact quaternionic symmetric spaces $M^{*}$}

Let $M^{*}$ be a compact quaternionic symmetric space. Then it is simply connected and irreducible. Moreover let $N^{*}$ be a complete totally complex totally geodesic submanifold such that $4 \operatorname{dim} N^{*}=\operatorname{dim} M^{*}$. Then $N^{*}$ is a substantial symmetric submanifold. Moreover the effective COQ $(\overline{\mathrm{g}}, \sigma, \tau,\langle\rangle)$ associated with $(M, N)$ satisfies the conditions (C.1), (C.2). In fact, it holds $\operatorname{dim} \overline{\mathfrak{a}}=1$ and the subalgebra $\boldsymbol{R} \cdot 1_{\bar{p}^{*}} \oplus\left[\operatorname{ad}_{\bar{p}^{*}}(\overline{\mathfrak{a}}), J\right] \oplus \boldsymbol{R} \cdot J \oplus \operatorname{ad}_{\bar{p}^{*}}(\overline{\mathfrak{a}})$ of End $\left(\bar{p}^{*}\right)$ gives the quaternionic structure of $M^{*}$ at the base point $o^{*}$, where End $\left(\overline{\mathfrak{p}}^{*}\right)$ denotes the associative algebra of all endomorphisms of $\bar{p}^{*}$. We refer to Takeuchi [20] for the definitions, the classifications, and the above notes of quaternionic symmetric space and totally complex totally geodesic submanifold. In this paper we just list up all COQ's $(\overline{\mathfrak{g}}, \sigma$, $\tau,<>$ ) of this type in Table I. Then, from the table, we can see that they, except No. 1, satisfy

(C.3) $\overline{\mathfrak{f}}_{+}=\left[\overline{\mathfrak{p}}_{+}, \overline{\mathfrak{p}}_{+}\right]$and thus $\overline{\mathfrak{t}}^{*}=\overline{\mathfrak{h}}_{+} \oplus \overline{\mathfrak{p}}_{+}$.

TheOREM 5.4. Assume that $(\overline{\mathrm{g}}, \sigma, \tau,\langle>)$ is an effective COQ in Table I, except No. 1 , No. 2. Let $S$ be a substantial symmetric submanifold with the associated $N$. Then $(M, S)$ is equivalent to $(M, N)$ and particularly $S$ is totally geodesic.

PROOF. Note that $M^{*}$ is an irreducible riemannian symmetric space which is not hermitian, except No. 1, No. 2. For such an $M^{*}$ it is scalar, an endomorphism $\Sigma$ of $\bar{p}^{*}$ such that $\left[\Sigma, \operatorname{ad}_{\bar{p}^{*}}(T)\right]=0$ for $T \in \overline{\mathfrak{f}}^{*}$. Now let $(\overline{\mathfrak{g}}, \sigma, \tau,\langle>: \lambda)$ be the $Q \Lambda$-pair associated with $(M, S)$. 
Then $\Lambda_{\lambda}$ satisfies the above condition for $\Sigma$ by the condition (C.3) and Lemma 5.3. Hence it is scalar and, since it is skew symmetric, it follows $\Lambda_{\lambda}=0$. This implies our claim. q.e.d.

REMARK 5.5. The effective COQ's of No. 2 appear in Table II again. Hence $Q \Lambda$-pairs associated with the COQ's will be looked up in Part II. Next consider the effective COQ's in No. 1. Then $(M, N)$ is equivalent to $\left(G_{i, n-i}(C), C P_{i-1} \times C P_{n-i-1}\right)$, where $G_{i, n-i}(C)$ denotes the Grassmann manifold of $i$-subspaces in $C^{n}$. Particularly if $i=1,(M, N)$ is equivalent to $\left(C P_{n-1}, C P_{n-2}\right)$. Then symmetric submanifolds $S$ have already been classified. In fact, $S$ are equivalent to either of the linear subspace $C P_{n-2}$ or the complex quadric $C Q_{n-2}$. For the case when $i \geqq 2, S$ is equivalent to the totally geodesic submanifold $\boldsymbol{C P _ { i - 1 }} \times \boldsymbol{C} P_{n-\mathrm{i}-1}$. In fact, note that $S$ is also a parallel Kahlerian submanifold. Such submanifolds of hermitian symmetric spaces have been classified by Tsukada [23]. By the classification it follows that $S$ is equivalent to $C P_{i-1} \times C P_{n-\mathrm{i}-1}$.

\section{Totally real totally geodesic symmetric submanifolds $N^{*}$ of compact hermitian symmetric spaces $M^{*}$}

Let $M^{*}$ be a compact simply connected hermitian symmetric space and $N^{*}$ a complete totally real totally geodesic submanifold of $M^{*}$ such that $2 \operatorname{dim} N^{*}=\operatorname{dim} M^{*}$. Then $N^{*}$ is a substantial symmetric submanifold. Moreover the effective $\operatorname{COQ}(\overline{\mathfrak{g}}, \sigma, \tau,\langle\rangle)$ satisfies the conditions (C.1), (C.2). In fact, it holds $\overline{\mathfrak{a}}=\{0\}$ and $H_{0} \in \mathrm{C}\left(\overline{\mathfrak{f}}^{*}\right) \cap \overline{\mathfrak{p}}_{+} \neq\{0\}$, where $\mathrm{C}\left(\overline{\mathfrak{f}}^{*}\right)$ denotes the center of $\overline{\mathfrak{f}}^{*}$. If $(\overline{\mathfrak{g}}, \sigma, \tau,\langle\rangle)$ is irreducible, it holds $\operatorname{dim}\left(\mathrm{C}\left(\overline{\mathfrak{f}}^{*}\right) \cap \overline{\mathfrak{p}}_{+}\right)=1$ and we may suppose that $J$ gives the complex structure of $M^{*}$ at $o^{*}$. We refer to Takeuchi [19] for the definitions, the classifications, and the above notes of hermitian symmetric space and totally real totally geodesic submanifold. In this paper we just list up all irreducible COQ's $(\overline{\mathrm{g}}, \sigma, \tau,\langle>)$ of this type in Table II.

Assume that the effective $\operatorname{COQ}(\overline{\mathfrak{g}}, \sigma, \tau,\langle>)$ is irreducible. For $c \in \boldsymbol{R}$ define a linear map $\lambda_{c}$ of $\overline{\mathfrak{p}}_{-}$to $\overline{\mathfrak{f}}_{-}$by $\lambda_{c}=c J$. Then $\lambda_{c}$ satisfies the conditions ( $\left.\Lambda .1\right),(\Lambda .2)$. In fact, they follow from the fact that $H_{0} \in \mathrm{C}\left(\overline{\mathfrak{f}}^{*}\right)$. Hence $\left(\overline{\mathrm{g}}, \sigma, \tau,\left\langle>: \lambda_{c}\right)\right.$ is a $Q \Lambda$-pair. Denote by $\left(M, S_{c}\right)$ the pair associated with the $Q \Lambda$-pair.

LEMma 5.6. (1) The submanifold $S_{c}$ is pseudo-umbilical. Moreover, (2) symmetric submanifolds $S_{c}, S_{-c}$ are equivalent to each other. And symmetric submanifolds $S_{c}, c \geqq 0$, are not equivalent to one another.

Proof. (1) Since $S_{c}$ is equivariant, we may show that it is pseudo-umbilical at the base point $o$. Put $\mathfrak{m}_{\mathrm{c}}=\left\{X+c J X ; X \in \overline{\mathfrak{p}}_{-}\right\}$and identify $T_{o} S_{c}, N_{o} S_{c}$ with $\mathfrak{m}_{c}, \overline{\mathfrak{p}}_{+}$respectively. Then the curvature tensor $R_{o}$ and the second fundamental form $\alpha_{o}$ of $S_{c}$ at $o$ are identified as follows: 


$$
\begin{aligned}
R_{o}(X+c J X, Y+c J Y)(Z+c J Z) & =-[[X+c J X, Y+c J Y], Z+c J Z] \\
& =\left(1+c^{2}\right)(-[[X, Y], Z]-c J([[X, Y], Z]))
\end{aligned}
$$

and

$$
\alpha_{o}(X+c J X, Y+c J Y)=[X, c J Y]
$$

for $X, Y, Z \in \overline{\mathfrak{p}}_{-}$. (cf. See Naitoh [11] for $\alpha_{0}$.) Moreover identify $\mathfrak{m}_{c}$ with $\overline{\mathfrak{p}}_{-}$by the correspondence: $\mathfrak{m}_{c} \ni X+c J X \leftrightarrow X \in \overline{\mathfrak{p}}_{-}$. Then $R_{o}, \alpha_{o}$ are also identified as follows:

$$
R_{o}(X, Y) Z=-\left(1+c^{2}\right)[[X, Y], Z], \quad \alpha_{o}(X, Y)=[X, c J Y]
$$

for $X, Y, Z \in \overline{\mathfrak{p}}_{-}$. Denote by $\eta_{o}$ the mean curvature vector of $S_{c}$ at $o$ and by $\bar{R}_{o}$ the curvature tensor of $M$ at $o$. Moreover denote by $R_{\sigma^{*}}^{*}, R i c_{o^{*}}^{*}$ the curvature tensor, the Ricci tensor of $M^{*}$ at $o^{*}$ respectively. Take an orthonormal basis $\left\{e_{1}, \cdots, e_{s}\right\}$ of $\overline{\mathfrak{p}}_{-}$. Then, by (5.3) and the Gauss' equation, it follows

$$
\begin{aligned}
s\left\langle\alpha_{o}(X, Y), \eta_{o}\right\rangle= & \sum_{i}\left(\left\langle\bar{R}_{o}\left(X, e_{i}\right) Y, e_{i}\right\rangle-\left\langle R_{o}\left(X, e_{i}\right) Y, e_{i}\right\rangle+\left\langle\alpha_{o}\left(X, e_{i}\right), \alpha_{o}\left(Y, e_{i}\right)\right\rangle\right) \\
& =c^{2} \sum_{i}\left(\left\langle\left[\left[X, e_{i}\right], Y\right], e_{i}\right\rangle+\left\langle\left[X, J e_{i}\right],\left[Y, J e_{i}\right]\right\rangle\right) \\
& =-c^{2} \sum_{i}\left(\left\langle R_{o}^{*}\left(X, e_{i}\right) Y, e_{i}\right\rangle+\left\langle R_{o^{*}}^{*}\left(X, J e_{i}\right) Y, J e_{i}\right\rangle\right) \\
& =c^{2} \operatorname{Ric}_{o^{*}}^{*}(X, Y)
\end{aligned}
$$

for $X, Y \in \overline{\mathfrak{p}}_{-}$. Note that $M^{*}$ is Einstein. In fact, for No. $7 \sim$ No. $18, M^{*}$ is irreducible and thus Einstein. For No. $1 \sim$ No. $6, M^{*}$ is not irreducible, but \langle\rangle is left invariant by $\sigma$. Hence $M^{*}$ is Einstein. Consequently it follows $\left\langle\alpha_{o}(X, Y ;), \eta_{o}\right\rangle=a\langle X, Y\rangle$ for some $a \in R$. This implies that $S_{c}$ is pseudo-umbilical at $o$.

(2) The isomorphism $\sigma$ of $(\overline{\mathfrak{g}}, \sigma, \tau,\langle\rangle)$ satisfies $\sigma\left(\mathfrak{m}_{c}\right)=\mathfrak{m}_{-c}$. Hence $S_{c}$ is equivalent to $S_{-c}$.

The second claim is obvious by (5.3).

q.e.d.

THEOREM 5.7. Assume that $(\overline{\mathrm{g}}, \sigma, \tau,<>)$ is an effective COQ in Table II, except No. 13, $i=1$. Let $S$ be a substantial symmetric submanifold with the associated $N$. Then $(M, S)$ is equivalent to some $\left(M, S_{c}\right)$.

Proof. Assume that $(\overline{\mathfrak{g}}, \sigma, \tau,\langle\rangle)$ is one of No. $7 \sim$ No. 18 , except No. $13, i=1$. Then $M^{*}$ is an irreducible hermitian symmetric space and satisfies the condition (C.3). Let ( $\overline{\mathfrak{g}}$, $\sigma, \tau,\langle>: \lambda)$ be the $Q \Lambda$-pair associated with $(M, S)$. Then it follows that $\left[\Lambda_{\lambda}, \operatorname{ad}_{\bar{p}^{*}}(T)\right]=0$ for $T \in \overline{\mathfrak{f}}^{*}$ by Lemma 5.3. Generally for an irreducible hermitian symmetric space, it is a scalar multiple of the complex structure $J$, a skew symmetric endormorphism $\Sigma$ of $\overline{\mathfrak{p}}^{*}$ such that $\left[\Sigma, \operatorname{ad}_{\mathfrak{p}^{*}}(T)\right]=0$ for $T \in \overline{\mathfrak{f}}^{*}$. Hence it holds $\Lambda_{\lambda}=c J$ for some $c \in \boldsymbol{R}$. This implies that $\lambda=c J$. Hence $(M, S)$ is equivalent to $\left(M, S_{c}\right)$. 
Next assume that $(\overline{\mathfrak{g}}, \sigma, \tau,\langle>)$ is one of No. $1 \sim$ No. 6 . Then the COQ is constructed as follows. Let $\left(\hat{\mathfrak{g}}, \hat{\tau},\left\langle^{\wedge}\right\rangle\right)$ be an irreducible orthogonal symmetric Lie algebra corresponding to an irreducible hermitian symmetric space of compact type. Moreover let $\hat{\mathfrak{g}}=\hat{\mathfrak{l}} \oplus \hat{\mathfrak{p}}$ be the eigenspace decomposition by $\hat{\tau}$ into the $(+1)$-eigenspace $\hat{\mathfrak{f}}$ and the $(-1)$-eigenspace $\hat{\mathfrak{p}}$. Then $\hat{\mathfrak{t}}$ has the one-dimensional center $\mathrm{c}$ and is also decomposed into the sum of $\mathrm{c}$ and semisimple ideal $\mathfrak{f}^{\prime}$. Put $\overline{\mathfrak{g}}=\hat{\mathfrak{g}} \oplus \hat{\mathfrak{g}}$. The involutive automorphisms $\sigma, \tau$ and the inner product $\langle>$ are defined as follows:

$$
\begin{aligned}
& \sigma(X, Y)=(Y, X), \tau(X, Y)=(\hat{\tau}(X), \hat{\tau}(Y)), \\
& \langle(X, Y),(Z, W)\rangle=\langle\widehat{X, Z}\rangle+\langle\hat{Y, W}\rangle
\end{aligned}
$$

for $X, Y, Z, W \in \hat{\mathfrak{g}}$. Moreover $\overline{\mathfrak{f}}, \overline{\mathfrak{f}}_{ \pm}, \overline{\mathfrak{p}}, \overline{\mathfrak{p}}_{ \pm}$are given in the followings.

$$
\begin{array}{ll}
\overline{\mathfrak{f}}=\{(X, X) ; X \in \hat{\mathfrak{g}}\}, & \overline{\mathfrak{p}}=\{(X,-X) ; X \in \hat{\mathfrak{g}}\}, \\
\overline{\mathfrak{f}}_{+}=\{(X, X) ; X \in \hat{\mathfrak{f}}\}, & \overline{\mathfrak{p}}_{+}=\{(X,-X) ; X \in \hat{\mathfrak{f}}\}, \\
\overline{\mathfrak{f}}_{-}=\{(X, X) ; X \in \hat{\mathfrak{p}}\}, & \overline{\mathfrak{p}}_{-}=\{(X,-X) ; X \in \hat{\mathfrak{p}}\} .
\end{array}
$$

Now let $(\overline{\mathfrak{g}}, \sigma, \tau,\langle>: \lambda)$ be the $Q \Lambda$-pair associated with $(M, S)$ and define an endomorphism $\hat{\lambda}$ of $\hat{\mathfrak{p}}$ such that $\lambda(X,-X)=(\hat{\lambda}(X), \hat{\lambda}(X))$ for $X \in \hat{\mathfrak{p}}$. Note that $\lambda^{t}(X, X)=\left(\hat{\lambda}^{t}(X)\right.$, $\left.-\hat{\lambda}^{t}(X)\right)$ for $X \in \hat{\mathfrak{p}}$. Then by (5.1), (5.2) it follows

$$
\operatorname{ad}_{\hat{\mathfrak{p}}}(T) \circ \hat{\lambda}=-\hat{\lambda}^{t} \circ \operatorname{ad}_{\hat{\mathfrak{p}}}(T), \quad \hat{\lambda} \circ \operatorname{ad}_{\hat{\mathfrak{p}}}(T)=-\operatorname{ad}_{\hat{\mathfrak{p}}}(T) \circ \hat{\lambda}^{t}
$$

for $T \in \hat{\mathfrak{l}}$. Hence the endomorphisms $\hat{\Lambda}_{ \pm}$of $\hat{\mathfrak{p}}$ defined by $\hat{\Lambda}_{ \pm}=\left(\hat{\lambda} \pm \hat{\lambda}^{t}\right) / 2$ satisfy

$$
\operatorname{ad}_{\hat{p}}(T) \circ \Lambda_{ \pm}=\mp \Lambda_{ \pm} \circ \operatorname{ad}_{\hat{p}}(T)
$$

for $T \in \hat{\mathfrak{t}}$. Note that the complex structure $\hat{J}$ of $\left(\hat{\mathfrak{g}}, \hat{\tau},\left\langle^{\wedge}\right\rangle\right)$ is given by $\operatorname{ad}_{\hat{\mathfrak{p}}}\left(\hat{H}_{0}\right)$ for some $\hat{H}_{0} \in$ c. Since $\hat{\Lambda}_{-}$is skew symmetric, it follows $\hat{\Lambda}_{-}=c \hat{J}$ for some $c \in \boldsymbol{R}$ in the same way as above. Also it holds $\operatorname{ad}_{\hat{\mathfrak{p}}}(T) \circ \hat{\Lambda}_{+}^{2}=\hat{\Lambda}_{+}^{2} \circ \operatorname{ad}_{\hat{\mathfrak{p}}}(T)$ for $T \in \hat{\mathfrak{f}}$ by $(5.4)$. Hence $\hat{\Lambda}_{+}^{2}$ is scalar, since it is symmetric and $\left(\hat{\mathfrak{p}}, \hat{\tau},\left\langle^{\prime}\right\rangle\right)$ is irreducible. On the other hand, since $\mathfrak{t}^{\prime}=[\hat{\mathfrak{t}}, \hat{\mathfrak{t}}]$, it follows $\operatorname{ad}_{\hat{\mathfrak{p}}}(T) \circ \hat{\Lambda}_{+}=\hat{\Lambda}_{+} \circ \operatorname{ad}_{\hat{\mathfrak{p}}}(T)$ for $T \in \mathfrak{f}^{\prime}$ and thus $\left[\mathfrak{f}^{\prime}, \hat{\Lambda}_{+}(\hat{\mathfrak{p}})\right]=\{0\}$ by (5.4). Here note that $\mathfrak{f}^{\prime} \neq\{0\}$. If $\hat{\Lambda}_{+}$is non-degenerate, it follows $\left[\mathfrak{f}^{\prime}, \hat{\mathfrak{p}}\right]=\{0\}$. This contradicts that the adjoint representation $\operatorname{ad}_{\hat{\mathfrak{p}}}$ of $\hat{\mathfrak{t}}$ is faithful. Hence it holds $\hat{\Lambda}_{+}=0$. This implies that $\hat{\lambda}=c \hat{\jmath}$. Since we may suppose that the complex structure $J$ of $\bar{p}^{*}$ is given by $\operatorname{ad}_{\bar{p}^{*}}\left(H_{0},-H_{0}\right)$, it follows $\lambda=c J$. Hence $(M, S)$ is equivalent to $\left(M, S_{c}\right)$.

REMARK 5.8. If $(\bar{g}, \sigma, \tau,\langle\rangle)$ is one of No. $13, i=1$, the pairs $\left(M^{*}, N^{*}\right),(M, N)$ are equivalent to $\left(C Q_{n}, S^{n}\right),\left(S^{n+1}, S^{n}\right)$ for some $n$ respectively. Hence symmetric submanifolds with the associated $N$ have already been classified. In fact, they are equivalent to generalized Clifford torus of $S^{n+1}$.

REMARK 5.9. The symmetric submanifolds $\left(M, S_{c}\right)$ associated with $(M, N)$ in No. $1 \sim$ No. 6 , have been constructed in Tsukada [23]. 


\section{References}

[1] Backes, E. and Reckziegel, H., On symmetric submanifolds of spaces of constant curvature, Math. Ann. 263 (1983), 419-433.

[2] Berger, M, Les espaces symetriques non compacts, Ann. Sci. Ecole Norm. Sup. (4), 74 (1957), . 85-177.

[3] Chen, B.Y. and Nagano, T., Totally geodesic submanifolds of symmetric spaces II, Duke Math. J., 45 (1978), 405-425.

[4] Ferus, D., Symmetric submanifolds of euclidean space, Math. Ann., 247 (1980), 81-93.

[5] Funabashi, S., Totally complex submanifolds of a quaternionic Kaehlerian manifold, Kodai Math. J., 2 (1979), 314-336.

[6] Helgason, S., Differential Geometry, Lie groups and Symmetric spaces, Academic Press, New York, 1978.

[7] Kobayashi, S. and Nagano, T. On filtered Lie algebras and geometric structures I, J. Math. and Mech., 13 (1964), 875-907.

[8] Kobayashi, S. and Nomizu, K., Fundations of Differential Geometry I, II, Interscience, New York, 1963, 1969.

[9] Kon, M., On some complex submanifolds in Kaehler manifolds, Canad. J. Math., 26 (1974), 1442-1449.

[10] Nakagawa, H. and Takagi, R., On locally symmetric Kaehler submanifolds in a complex projective space, J. Math. Soc. Japan, 28 (1976), 638-667.

[11] Naitoh, H., Isotropic submanifolds with parallel second fundamental form in $P^{m}(c)$, Osaka J. Math., 18 (1981), 427-464.

[12] - Totally real parallel submanifolds in $P^{n}(c)$, Tokyo J. of Math., 4 (1981), 279-306.

[13] - Parallel submanifolds of complex space forms I, II, Nagoya Math. J., 90 (1983), 85-117 and 91 (1983), 119-149.

[14] Naitoh, H. and Takeuchi, M., Totally real submanifolds and symmetric bounded domains, Osaka J. Math., 19 (1982), 717-731.

[15] Naitoh, H., Symmetric submanifolds of compact symmetric spaces, Lect. Notes in Math., 1090 (Proceedings), ed. by K. Kenmotsu, Springer-Verlag, New York, 1984.

[16] Strübing, W., Symmetric submanifolds of riemannian manifolds, Math. Ann., 245 (1979), 3744.

[17] Takeuchi, M., Parallel submanifolds of space forms, Manifolds and Lie groups, in honor of $Y$. Matsushima, ed. by J. Hano et al., Birkhauser, Boston, 1981, 429-447.

[18] - Parallel projective manifolds and symmetric bounded domains, Preprint.

[19] - Stability of certain minimal submanifolds of compact Hermitian symmetric spaces, Tohoku J. Math., 36 (1984), 293-314.

[20] - Totally complex submanifolds of quaternionic symmetric spaces, Preprint.

[21] Tsukada, K., Parallel submanifolds in a quaternion projective space, Preprint.

[22] - Parallel submanifolds of Cayley plane, Preprint.

[22] - Parallel Kaehler submanifolds of hermitian symmetric spaces, Preprint.

Department of Mathematics

Yamaguchi University

Yamaguchi, 753 Japan 
TABLE I

\begin{tabular}{|c|c|c|c|c|}
\hline No. & $(\hat{\mathfrak{g}}, \hat{\tau})$ & $(\overline{\mathfrak{g}}, \sigma), M$ & $(\overline{\mathfrak{g}}, \tau), M^{*}$ & Remark \\
\hline 1 & $\begin{array}{l}\mathfrak{B} \mathfrak{u}(i, n-\mathrm{i}) / \\
\quad \mathfrak{B} \mathfrak{u}(1,1)+\mathfrak{B} \mathfrak{u}(n-\mathrm{i}-1, i-1) \\
\quad+\boldsymbol{T}\end{array}$ & $\begin{array}{l}\mathfrak{g} \mathfrak{u}(n) / \\
\mathfrak{s}(\mathfrak{u}(i)+\mathfrak{u}(n-i))\end{array}$ & $\begin{array}{l}\mathfrak{g} \mathfrak{u}(n) / \\
\mathfrak{s}(\mathfrak{u}(2)+\mathfrak{u}(n-2))\end{array}$ & $\begin{array}{l}n \geqq 4 \\
1 \leqq i \leqq n-i \\
N^{*} \subset M^{*}: \text { Kahler }\end{array}$ \\
\hline 2 & $\begin{array}{l}\operatorname{sI}(n ; \boldsymbol{R}) / \\
\quad \operatorname{gl}(2 ; \boldsymbol{R})+\operatorname{sI}(n-2 ; \boldsymbol{R})+\boldsymbol{R}\end{array}$ & $\mathbb{8} \mathfrak{u}(n) / \mathfrak{s} \mathfrak{o}(n)$ & $\begin{array}{l}\mathfrak{s u}(n) / \\
\mathfrak{s}(\mathfrak{u}(2)+\mathfrak{u}(n-2))\end{array}$ & $\begin{array}{l}n \geqq 4, \\
N^{*} \subset M^{*}: \text { totally } \\
\quad \text { real }\end{array}$ \\
\hline 3 & $\begin{array}{l}80(i, n-i) / \\
80(2,2)+80(i-2, n-i-2)\end{array}$ & $\begin{array}{l}\mathfrak{g} \mathfrak{o}(n) / \\
\mathfrak{s o}(i)+\mathfrak{s} \mathfrak{0}(n-i)\end{array}$ & $\begin{array}{l}80(n) / \\
80(4)+80(n-4)\end{array}$ & $\begin{array}{l}n \geqq 7 \\
2 \leqq i \leqq n-i\end{array}$ \\
\hline 4 & $\begin{array}{l}\mathfrak{s p}(n ; \boldsymbol{R}) / \\
\mathbb{8 p}(1 ; \boldsymbol{R})+\mathfrak{s p}(n-1 ; \boldsymbol{R})\end{array}$ & $\mathbb{8} \mathfrak{p}(n) / \mathfrak{u}(n)$ & $\begin{array}{l}\mathfrak{s p}(n) / \\
\mathfrak{s p}(1)+\mathfrak{s p}(n-1)\end{array}$ & $n \geqq 3$ \\
\hline 5 & $\begin{array}{l}80^{*}(2 n) / \\
80^{*}(4)+80^{*}(2 n-4)\end{array}$ & $8 \mathfrak{s o}(2 n) / \mathfrak{u}(n)$ & $\begin{array}{l}\mathfrak{g} \mathfrak{0}(2 n) / \\
\mathfrak{8 0}(4)+\mathfrak{g} \mathfrak{0}(2 n-4)\end{array}$ & $n \geqq 5$ \\
\hline 6 & $\begin{array}{l}G_{2}^{*} / \\
\operatorname{sl}(2 ; \boldsymbol{R})+\mathfrak{g l}(2 ; \boldsymbol{R})\end{array}$ & $\mid \begin{array}{l}G_{2} / \\
\mathfrak{s u}(2)+\mathfrak{s} \mathfrak{u}(2)\end{array}$ & $\begin{array}{l}G_{2} / \\
\mathbf{s u}(2)+\mathfrak{z u}(2)\end{array}$ & \\
\hline 7 & $\begin{array}{l}F_{4}^{1} / \\
\mathbb{g p}(3 ; R)+\mathfrak{g l}(2 ; \boldsymbol{R})\end{array}$ & $\begin{array}{l}F_{4} / \\
\mathbb{8 p}(3)+\mathfrak{g u}(2)\end{array}$ & $\begin{array}{l}F_{4} / \\
\mathfrak{s p}(3)+\mathfrak{s u}(2)\end{array}$ & \\
\hline 8 & $\begin{array}{l}E_{6}^{1} / \\
\quad \mathfrak{g l}(6 ; \boldsymbol{R})+\operatorname{gl}(2 ; \boldsymbol{R})\end{array}$ & $E_{6} / 8 \mathfrak{p}(4)$ & $\begin{array}{l}E_{6} / \\
\mathbf{8 u}(6)+\mathfrak{z u}(2)\end{array}$ & \\
\hline 9 & $\begin{array}{l}E_{6}^{2} / \\
\mathfrak{s u}(3,3)+\mathfrak{s}(2 ; \boldsymbol{R})\end{array}$ & $\begin{array}{l}E_{6} / \\
\mathfrak{s u}(6)+\mathfrak{s} \mathfrak{u}(2)\end{array}$ & $\begin{array}{l}E_{6} / \\
\mathfrak{s u}(6)+\mathfrak{s u}(2)\end{array}$ & \\
\hline 10 & $\begin{array}{l}E_{6}^{3} / \\
\mathfrak{s u}(1,5)+\operatorname{sil}(2 ; \boldsymbol{R})\end{array}$ & $\begin{array}{l}E_{6} / \\
80(10)+T\end{array}$ & $\begin{array}{l}E_{6} / \\
\mathfrak{s} \mathfrak{u}(6)+\mathfrak{s u}(2)\end{array}$ & \\
\hline 11 & $\begin{array}{l}E_{7}^{1} / \\
\mathbf{B o}_{0}(6,6)+\mathfrak{g l}(2 ; R)\end{array}$ & $E_{7} / \mathfrak{\&} \mathfrak{u}(8)$ & $\begin{array}{l}E_{7} / \\
\mathfrak{s}_{0}(12)+\mathfrak{s u}(2)\end{array}$ & \\
\hline 12 & $\begin{array}{l}E_{7}^{2} / \\
80^{*}(12)+8 \mathrm{gl}(2 ; \boldsymbol{R})\end{array}$ & $\begin{array}{l}E_{7} / \\
\mathfrak{s o}(12)+\mathfrak{z} \mathfrak{u}(2)\end{array}$ & $\begin{array}{l}E_{7} / \\
8 \mathfrak{o}(12)+\mathfrak{g u}(2)\end{array}$ & \\
\hline 13 & $\begin{array}{l}E_{7}^{3} / \\
80(2,10)+8 \mathfrak{I}(2 ; \boldsymbol{R})\end{array}$ & $\stackrel{E_{7} /}{E_{6}+\boldsymbol{T}}$ & $\begin{array}{l}E_{7} / \\
80(12)+8 \mathfrak{u}(2)\end{array}$ & \\
\hline 14 & $\begin{array}{l}E_{8}^{1} / \\
E_{7}^{1}+8 \mathrm{gl}(2 ; \boldsymbol{R})\end{array}$ & $E_{8} / 80(16)$ & $\begin{array}{l}E_{8} / \\
E_{7}+8 \mathfrak{u}(2)\end{array}$ & \\
\hline 15 & $\begin{array}{l}E_{8}^{2} / \\
E_{7}^{3}+8 \mathfrak{s}(2 ; \boldsymbol{R})\end{array}$ & $\begin{array}{l}E_{8} / \\
E_{7}+\mathfrak{g} \mathfrak{u}(2)\end{array}$ & $\begin{array}{l}E_{8} / \\
E_{7}+\mathfrak{s} \mathfrak{u}(2)\end{array}$ & \\
\hline
\end{tabular}


TABLE I (Continuation)

\begin{tabular}{|c|c|c|c|}
\hline No. & $\overline{\mathfrak{t}}_{+}$ & $\overline{\mathfrak{p}}_{-}\left(N, N^{*}\right), \overline{\mathfrak{f}}_{-}\left(N^{* \perp}\right)$ & $\overline{\mathbf{p}}_{+}\left(N^{\perp}\right)$ \\
\hline 1 & $\begin{array}{l}\mathfrak{g}(\mathfrak{u}(1)+\mathfrak{u}(1))+ \\
\quad \mathfrak{g}(\mathfrak{u}(i-1)+\mathfrak{u}(n-i-1))+T\end{array}$ & $\begin{array}{l}\mathfrak{g} \mathfrak{u}(i) / \mathfrak{s}(\mathfrak{u}(1)+\mathfrak{u}(i-1)) \\
\quad+ \\
\mathfrak{B} \mathfrak{u}(n-i) / \mathfrak{z}(\mathfrak{u}(1)+\mathfrak{u}(n-i-1))\end{array}$ & $\begin{array}{l}\mathbf{g} \mathfrak{u}(2) / \mathfrak{g}(\mathfrak{u}(1)+\mathfrak{u}(1)) \\
\quad+8 \mathfrak{u}(n-i) / \mathfrak{g}(\mathfrak{u}(1)+\mathfrak{u}(n-i-1))\end{array}$ \\
\hline 2 & $80(2)+80(n-2)$ & $\begin{array}{l}80(n) / \\
80(2)+80(n-2)\end{array}$ & $\begin{array}{l}8 \mathfrak{z}(2) / 8 \mathfrak{o}(2) \\
+8 \mathfrak{u}(n-2) / 8 \mathfrak{o}(n-2)+T\end{array}$ \\
\hline 3 & $\begin{array}{l}80(2)+80(2)+80(i-2)+ \\
80(n-i-2)\end{array}$ & $\begin{array}{l}80(i) / 80(2)+80(i-2) \\
+80(n-i) / 80(2)+80(n-i-2)\end{array}$ & $\begin{array}{l}80(4) / 80(2)+80(2) \\
+80(n-4) / 80(i-2)+80(n-i-2)\end{array}$ \\
\hline 4 & $\mathfrak{u}(1)+\mathfrak{u}(n-1)$ & $\begin{array}{l}\mathfrak{u}(n) / \\
\mathfrak{u}(1)+\mathfrak{u}(n-1)\end{array}$ & $\begin{array}{l}\mathfrak{B p}(1) / \mathfrak{u}(1) \\
\quad+\mathfrak{s p}(n-1) / \mathfrak{u}(n-1)\end{array}$ \\
\hline 5 & $\mathfrak{u}(2)+\mathfrak{u}(n-2)$ & $\begin{array}{l}\mathfrak{u}(n) / \\
\mathfrak{u}(2)+\mathfrak{u}(n-2)\end{array}$ & $\begin{array}{l}80(4) / \mathfrak{u}(2) \\
+8 \mathfrak{o}(2 n-4) / \mathfrak{u}(n-2)\end{array}$ \\
\hline 6 & $80(2)+80(2)$ & $8 \mathfrak{B}(2) / \mathfrak{8} \mathfrak{o}(2)+\mathfrak{8} \mathfrak{u}(2) / \mathscr{8} \mathfrak{o}(2)$ & $8 \mathfrak{u}(2) / \mathfrak{8} \mathfrak{o}(2)+\mathfrak{s} \mathfrak{u}(2) / 8 \mathfrak{s}(2)$ \\
\hline 7 & $\mathfrak{u}(3)+8 \mathfrak{s}(2)$ & $8 \mathfrak{p}(3) / \mathfrak{u}(3)+8 \mathfrak{u}(2) / 8 \mathfrak{s}(2)$ & $8 \mathfrak{p}(3) / \mathfrak{u}(3)+8 \mathfrak{u}(2) / 8 \mathfrak{o}(2)$ \\
\hline 8 & $80(6)+80(2)$ & $8 p(4) / \mathfrak{u}(4)$ & $8 \mathfrak{B}(6) / \mathfrak{8 0}(6)+8 \mathfrak{u}(2) / 8 \mathfrak{o}(2)$ \\
\hline 9 & $\mathfrak{z}(\mathfrak{u}(3)+\mathfrak{u}(3))+\mathfrak{s o}(2)$ & $\begin{array}{l}\mathbf{g u}(6) / \mathfrak{g}(\mathfrak{u}(3)+\mathfrak{u}(3)) \\
\quad+\boldsymbol{g} \mathfrak{u}(2) / \mathfrak{8} \mathfrak{o}(2)\end{array}$ & $\begin{array}{l}\mathbf{8} \mathfrak{u}(6) / \mathfrak{g}(\mathfrak{u}(3)+\mathfrak{u}(3)) \\
\quad+\boldsymbol{8} \mathfrak{u}(2) / \mathfrak{8 0}(2)\end{array}$ \\
\hline 10 & $\mathfrak{z}(\mathfrak{u}(1)+\mathfrak{u}(5))+\mathfrak{s o}(2)$ & $8 \mathfrak{s o}(10) / \mathfrak{u}(5)$ & $\begin{array}{l}\mathfrak{g} \mathfrak{u}(6) / \mathfrak{z}(\mathfrak{u}(1)+\mathfrak{u}(5)) \\
\quad+\mathfrak{z} \mathfrak{u}(2) / \mathfrak{\&} \mathfrak{o}(2)\end{array}$ \\
\hline 11 & $80(6)+80(6)+80(2)$ & $\mathfrak{s} \mathfrak{u}(8) / \mathfrak{g}(\mathfrak{u}(4)+\mathfrak{u}(4))$ & $\begin{array}{l}8 \mathfrak{o}(12) / 8 \mathfrak{g}(6)+8 \mathfrak{o}(6) \\
+\mathfrak{8 u}(2) / \mathfrak{8 0}(2)\end{array}$ \\
\hline 12 & $\mathfrak{u}(6)+80(2)$ & $\begin{array}{l}\mathbf{s o}(12) / \mathfrak{u}(6) \\
\quad+\mathbf{8} \mathfrak{u}(2) / \mathfrak{s o}(2)\end{array}$ & $\begin{array}{l}\mathbf{8 0}(12) / \mathfrak{u}(6) \\
\quad+\boldsymbol{8} \mathfrak{u}(2) / 8 \mathfrak{8}(2)\end{array}$ \\
\hline 13 & $80(2)+80(10)+80(2)$ & $\begin{array}{l}E_{6} / \\
\quad \mathfrak{8 0}(10)+\mathfrak{8 0}(2)\end{array}$ & $\begin{array}{l}8 \mathfrak{o}(12) / 8 \mathfrak{g}(2)+8 \mathfrak{o}(10) \\
+8 \mathfrak{u}(2) / 8 \mathfrak{o}(2)\end{array}$ \\
\hline 14 & $8 \mathfrak{u}(8)+8 \mathfrak{o}(2)$ & $80(16) / \mathfrak{u}(8)$ & $E_{7} / \mathfrak{s} \mathfrak{u}(8)+\mathfrak{s u}(2) / 8 \mathfrak{o}(2)$ \\
\hline 15 & $E_{6}+T+80(2)$ & $E_{7} / E_{6}+T+8 \mathfrak{u}(2) / 8 \mathfrak{o}(2)$ & $E_{7} / E_{6}+T+8 \mathfrak{u}(2) / 8 \mathfrak{o}(2)$ \\
\hline
\end{tabular}


TABLE II

\begin{tabular}{|c|c|c|c|c|}
\hline No. & $(\hat{\mathfrak{g}}, \hat{\tau})$ & $(\overline{\mathfrak{g}}, \sigma), M$ & $(\overline{\mathfrak{g}}, \tau), M^{*}$ & Remark \\
\hline 1 & $\begin{array}{l}\operatorname{sl}(n ; C) / \\
\operatorname{sl}(i ; C)+\operatorname{sl}(n-i ; C)+C^{*}\end{array}$ & $\begin{array}{l}\mathfrak{s} \mathfrak{u}(n)+\mathfrak{s} \mathfrak{u}(n) / \\
\mathfrak{s} \mathfrak{u}(n)\end{array}$ & $\begin{array}{l}\mathfrak{g} \mathfrak{u}(n) / \mathfrak{s}(\mathfrak{u}(i)+\mathfrak{u}(n-i)) \\
+ \\
\mathfrak{s} \mathfrak{u}(n) / \mathfrak{s}(\mathfrak{u}(i)+\mathfrak{u}(n-i))\end{array}$ & $\begin{array}{l}1 \leqq i \leqq n-\mathrm{i} \\
n \geqq 3\end{array}$ \\
\hline 2 & $\begin{array}{l}8 \mathfrak{s o}(2 n ; C) / \\
8 \mathfrak{l}(n ; C)+C^{*}\end{array}$ & $\begin{array}{r}80(2 n)+80(2 n) / \\
80(2 n)\end{array}$ & $\begin{array}{l}8 \mathfrak{D}(2 n) / \mathfrak{u}(n) \\
\quad+8 \mathfrak{D}(2 n) / \mathfrak{u}(n)\end{array}$ & $n \geqq 4$ \\
\hline 3 & $\begin{array}{l}80(n ; C) / \\
80(n-2 ; C)+C^{*}\end{array}$ & $\begin{array}{r}80(n)+80(n) / \\
80(n)\end{array}$ & $\begin{array}{l}\mathbb{8 0}(n) / 8 \mathfrak{B}(n-2)+T \\
+ \\
80(n) / 8 \mathfrak{B D}(n-2)+T\end{array}$ & $n \geqq 5, \neq 6$ \\
\hline 4 & $\begin{array}{l}\mathfrak{g} \mathfrak{p}(n ; C) / \\
\mathfrak{g l}(n ; C)+C^{*}\end{array}$ & 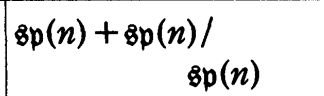 & $\begin{array}{l}\mathbb{s p}(n) / \mathfrak{u}(n) \\
\quad+\mathbb{s p}(n) / \mathfrak{u}(n)\end{array}$ & $n \geqq 3$ \\
\hline 5 & $\begin{array}{l}E_{6}^{C} / \\
80(10 ; C)+C^{*}\end{array}$ & $\begin{array}{r}E_{6}+E_{6} / \\
E_{6}\end{array}$ & $\begin{array}{l}E_{6} /{ }_{80}(10)+T \\
+E_{6} / \\
\mathbb{8 0}(10)+T\end{array}$ & \\
\hline 6 & $\begin{array}{l}E_{7}^{C} / \\
E_{6}^{C}+C^{*}\end{array}$ & $\begin{array}{r}E_{7}+E_{7} / \\
E_{7}\end{array}$ & ${ }^{E_{7} /} E^{6}+\boldsymbol{T}+E_{7} / E^{6}+\boldsymbol{T}$ & \\
\hline 7 & $\begin{array}{l}\mathfrak{g} \mathfrak{u}(n, n) / \\
\mathfrak{g l}(n ; C)+\boldsymbol{R}\end{array}$ & $\begin{array}{l}\mathfrak{s} \mathfrak{u}(2 n) / \\
\mathfrak{s}(\mathfrak{u}(n)+\mathfrak{u}(n))\end{array}$ & $\begin{array}{l}\boldsymbol{g} \mathfrak{u}(2 n) / \\
\mathfrak{z}(\mathfrak{u}(n)+\mathfrak{u}(n))\end{array}$ & $n \geqq 2$ \\
\hline 8 & $\begin{array}{l}80^{*}(4 n) / \\
8 \mathfrak{u}^{*}(2 n)+R\end{array}$ & $80(4 n) / \mathfrak{u}(2 n)$ & $80(4 n) / \mathfrak{u}(2 n)$ & $n \geqq 2$ \\
\hline 9 & $\begin{array}{l}\operatorname{gp}(n ; \boldsymbol{R}) / \\
\mathbb{g l}(n ; \boldsymbol{R})+\boldsymbol{R}\end{array}$ & $8 \mathfrak{p}(n) / \mathfrak{u}(n)$ & $\mathfrak{s p}(n) / \mathfrak{u}(n)$ & $n \geqq 2$ \\
\hline 10 & $\begin{array}{l}E_{7}^{3} / \\
E_{6}^{4}+R\end{array}$ & $\begin{array}{l}E_{7} / \\
E_{6}+\boldsymbol{T}\end{array}$ & $\begin{array}{l}E_{7} / \\
E_{6}+T\end{array}$ & \\
\hline 11 & $\begin{array}{l}\operatorname{sI}(n ; \boldsymbol{R}) / \\
\quad \operatorname{ll}(i ; \boldsymbol{R})+\operatorname{sI}(n-i ; \boldsymbol{R})+\boldsymbol{R}\end{array}$ & $\mathfrak{s} \mathfrak{u}(n) / \mathfrak{B o}(n)$ & $\begin{array}{l}\mathfrak{s u}(n) / \\
\mathfrak{s}(\mathfrak{u}(i)+\mathfrak{u}(n-i))\end{array}$ & $\begin{array}{l}1 \leqq i \leqq n-i \\
n \geqq 3\end{array}$ \\
\hline 12 & $\begin{array}{l}\mathfrak{g u}^{*}(2 n) / \\
\mathfrak{g u}^{*}(2 i)+\mathfrak{s u}^{*}(2 n-2 i)+\boldsymbol{R}\end{array}$ & $8 \mathfrak{z}(2 n) / \not \mathfrak{p} p(n)$ & $\begin{array}{l}\mathfrak{g u}(2 n) / \\
\mathbb{g}(\mathfrak{u}(2 i)+\mathfrak{u}(2 n-2 i))\end{array}$ & $\begin{array}{l}1 \leqq i \leqq n-i \\
n \geqq 3\end{array}$ \\
\hline 13 & $\begin{array}{l}80(i, n-i) / \\
80(i-1, n-i-1)+\boldsymbol{R}\end{array}$ & $\begin{array}{l}\mathbf{s 0}(n) / \\
80(i)+80(n-i)\end{array}$ & $\begin{array}{l}\mathbb{B} \mathfrak{0}(n) / \\
8 \mathfrak{D}(n-2)+T\end{array}$ & $\begin{array}{l}i=1: n \geqq 3, \\
i=2: n \geqq 7, \neq 6, \\
i=3: n \geqq 7, \\
i=4: n \geqq 9, \\
5 \leqq i \leqq n-\mathrm{i}\end{array}$ \\
\hline 14 & $\begin{array}{l}\operatorname{80}(n, n) / \\
\operatorname{gl}(n ; R)+R\end{array}$ & $\begin{array}{l}80(2 n) / \\
80(n)+80(n)\end{array}$ & $\mathbb{B D}(2 n) / \mathfrak{u}(n)$ & $n \geqq 4$ \\
\hline 15 & $\begin{array}{l}\mathfrak{s} \mathfrak{p}(n, n) / \\
\mathfrak{s u}^{*}(2 n)+\boldsymbol{R}\end{array}$ & $\begin{array}{l}\mathfrak{s p}(2 n) / \\
\mathfrak{s p}(n)+\mathfrak{s p}(n)\end{array}$ & $\mathbb{s p}(2 n) / \mathfrak{u}(2 n)$ & $n \geqq 2$ \\
\hline 16 & $\begin{array}{l}E_{6}^{1} / \\
80(5,5)+R\end{array}$ & $E_{6} / \mathfrak{B p}(4)$ & $\begin{array}{l}E_{6} / \\
\mathbf{8 0}(10)+T\end{array}$ & \\
\hline 17 & $\begin{array}{l}E_{6}^{4} / \\
80(1,9)+R\end{array}$ & $E_{6} / F_{4}$ & $\begin{array}{l}E_{6} / \\
\mathbf{s 0}(10)+T\end{array}$ & \\
\hline 18 & $\begin{array}{l}E_{7}^{1} / \\
E_{6}^{1}+R\end{array}$ & $E_{7} / \mathfrak{B} \mathfrak{u}(8)$ & $\begin{array}{l}E_{7} / \\
E_{6}+\boldsymbol{T}\end{array}$ & \\
\hline
\end{tabular}


Hiroo NAITOH

TABLE II (Continuation)

\begin{tabular}{|c|c|c|c|}
\hline No. & $\overline{\mathfrak{f}}_{+}$ & $\overline{\mathfrak{p}}_{-}\left(N, N^{*}\right), \overline{\mathfrak{t}}_{-}\left(N^{* \perp}\right)$ & $\overline{\mathbf{p}}_{+}\left(N^{\perp}\right)$ \\
\hline 1 & $\mathfrak{g}(\mathfrak{u}(i)+\mathfrak{u}(n-i))$ & $\mathfrak{s} \mathfrak{u}(n) / \mathfrak{s}(\mathfrak{u}(i)+\mathfrak{u}(n-i))$ & $\begin{array}{l}\mathfrak{s}(\mathfrak{u}(i)+\mathfrak{u}(n-i))+\mathfrak{z}(\mathfrak{u}(i) \\
+\mathfrak{u}(n-i)) / \\
\mathfrak{z}(\mathfrak{u}(i)+\mathfrak{u}(n-i))\end{array}$ \\
\hline 2 & $\mathfrak{u}(n)$ & $80(2 n) / \mathfrak{u}(n)$ & $\begin{array}{r}\mathfrak{u}(n)+\mathfrak{u}(n) / \\
\mathfrak{u}(n)\end{array}$ \\
\hline 3 & $80(n-2)+T$ & $\begin{array}{l}80(n) / \\
80(n-2)+T\end{array}$ & $\begin{array}{l}80(n-2)+T+80(n-2)+T / \\
80(n-2)+T\end{array}$ \\
\hline 4 & $\mathfrak{u}(n)$ & $\mathfrak{B p}(n) / \mathfrak{u}(n)$ & $\mathfrak{u}(n)+\mathfrak{u}(n) /_{\mathfrak{u}(n)}$ \\
\hline 5 & $80(10)+T$ & $\begin{array}{l}E_{6} / \\
80(10)+T\end{array}$ & $\begin{array}{l}80(10)+T+80(10)+T / \\
80(10)+T\end{array}$ \\
\hline 6 & $E_{6}+T$ & $\begin{array}{l}E_{7} / \\
E_{6}+T\end{array}$ & $\begin{array}{r}E_{6}+\boldsymbol{T}+E_{6}+\boldsymbol{T} / \\
E_{6}+\boldsymbol{T}\end{array}$ \\
\hline 7 & $\operatorname{sen}(n)$ & $T+\mathfrak{z} \mathfrak{u}(n)+\mathfrak{b u}(n) /_{\mathfrak{g} \mathfrak{u}(n)}$ & $T+\mathfrak{s} \mathfrak{u}(n)+\mathfrak{g} \mathfrak{u}(n) /_{\mathfrak{g} \mathfrak{u}(n)}$ \\
\hline 8 & $8 p(n)$ & $T+\varepsilon \mathfrak{u}(2 n) / \mathfrak{s p}(n)$ & $T+8 \mathfrak{u}(2 n) / \mathfrak{s p}(n)$ \\
\hline 9 & $80(n)$ & $T+8 \mathfrak{u}(n) / 8 \mathfrak{o}(n)$ & $T+8 \mathfrak{u}(n) / \mathbb{8} \mathfrak{0}(n)$ \\
\hline 10 & $F_{4}$ & $\boldsymbol{T}+E_{6} / F_{4}$ & $\boldsymbol{T}+E_{6} / F_{4}$ \\
\hline 11 & $80(i)+80(n-i)$ & $\begin{array}{l}80(n) / \\
80(i)+80(n-i)\end{array}$ & $\begin{array}{l}T+8 \mathfrak{u}(i) / 80(i) \\
+8 \mathfrak{s u}(n-i) / 80(n-i)\end{array}$ \\
\hline 12 & $8 \mathfrak{p}(i)+8 \mathfrak{p}(n-i)$ & $\stackrel{8 \mathfrak{p}(n) /}{8 \mathfrak{p}(i)+\mathbb{g} p(n-i)}$ & $\begin{array}{l}T+\mathfrak{s u}(2 i) / \mathfrak{s p}(i) \\
+\quad+\mathfrak{s u}(2 n-2 i) / \mathfrak{s p}(n-i)\end{array}$ \\
\hline 13 & $80(i-1)+80(n-i-1)$ & $\begin{array}{l}80(i) / 80(i-1) \\
+80(n-i) / 80(n-i-1)\end{array}$ & $\begin{array}{l}T+ \\
80(n-2) / 80(i-1)+80(n-i-1)\end{array}$ \\
\hline 14 & $80(n)$ & $80(n)+80(n) / 80(n)$ & $T+8 \mathfrak{u}(n) / 8 \mathfrak{o}(n)$ \\
\hline 15 & $8 p(n)$ & $\mathfrak{g p}(n)+\mathfrak{g p}(n) /_{\mathfrak{g p}(n)}$ & $T+8 \mathfrak{u}(2 n) / \mathfrak{s p}(n)$ \\
\hline 16 & $80(5)+80(5)$ & $8 \mathfrak{p}(4) / 8 \mathfrak{p}(2)+8 \mathfrak{p}(2)$ & $T+80(10) / 80(5)+80(5)$ \\
\hline 17 & $80(9)$ & $F_{4} / 80(9)$ & $T+80(10) / 80(9)$ \\
\hline 18 & $8 p(4)$ & $8 \mathfrak{u}(8) / \mathfrak{s p}(4)$ & $\boldsymbol{T}+E_{6} / 8 p(4)$ \\
\hline
\end{tabular}

